\title{
Phase Separation and Dynamics of Two-component Bose-Einstein Condensates
}

\author{
R. Navarro ${ }^{1}$, R. Carretero-González ${ }^{1, *}$, P.G. Kevrekidis ${ }^{2}$ \\ ${ }^{1}$ Nonlinear Dynamical Systems Group ${ }^{\dagger}$, Computational Science Research Center, \\ Department of Mathematics and Statistics, San Diego State University, San Diego CA, 92182-7720, USA \\ ${ }^{2}$ Department of Mathematics and Statistics, University of Massachusetts, Amherst, MA 01003-4515 USA
}

(Dated: Submitted to Phys. Rev. A, 2009)

\begin{abstract}
We study the interactions between two atomic species in a binary Bose-Einstein condensate to revisit the conditions for miscibility, oscillatory dynamics between the species, steady state solutions and their stability. By employing a variational approach for a quasi one-dimensional, two-atomic species, condensate we obtain equations of motion for the parameters of each species: amplitude, width, position and phase. A further simplification leads to a reduction of the dynamics into a simple classical Newtonian system where components oscillate in an effective potential with a frequency that depends on the harmonic trap strength and the interspecies coupling parameter. We develop explicit conditions for miscibility that can be interpreted as a phase diagram that depends on the harmonic trap's strength and the interspecies species coupling parameter. We numerically illustrate the bifurcation scenario whereby non-topological, phase-separated states of increasing complexity emerge out of a symmetric state, as the interspecies coupling is increased. The symmetry-breaking dynamical evolution of some of these states is numerically monitored and the associated asymmetric states are also explored.
\end{abstract}

\section{INTRODUCTION}

Over the past 15 years, the study of Bose-Einstein condensates (BECs) has gained a tremendous momentum, stemming from an intense and wide variety of theoretical, as well as experimental studies that have now been summarized in a number of books [1, 2]. One of the particularly intriguing aspects of the system is its effective nonlinearity stemming from a mean-field representation of the inter-atomic interactions. This, in turn, has led to a wide range of developments in the area of nonlinear matter waves in BECs [3] and the drawing of natural parallels between this field and that of nonlinear optics, where similar nonlinear Schrödinger (NLS) types of models arise [4].

One of the particularly interesting aspects of investigation of BECs within the realm of NLS (often referred to in the BEC context as Gross-Pitaevskii) equations is based on the examination of multi-component systems. Starting from the early work on ground-state solutions [5, 6] and small-amplitude excitations [7] of the order parameters, numerous investigations have been focused on the study of two hyperfine states or two different atomic species that can be condensed and confined concurrently. More specifically, a few among the numerous topics investigated involved the structure and dynamics of binary BECs [8, 9, 10], the formation of domain walls between immiscible species [8, 11], bound states of dark-dark [12], or dark-bright [13, 14], or coupled-vortex [15], or even spatially periodic states [16]. The early experimental efforts produced such binary mixtures of different hyperfine states of ${ }^{87} \mathrm{Rb}$ [17] and of ${ }^{23} \mathrm{Na}$ [19], but also of mixed

*URL: http://rohan.sdsu.edu/ rcarrete/

†URL: http://nlds.sdsu.edu condensates [18]. Efforts were later made to create twocomponent BECs with different atomic species, such as ${ }^{41} \mathrm{~K}-{ }^{87} \mathrm{Rb}[20]$ and ${ }^{7} \mathrm{Li}-{ }^{133} \mathrm{Cs}[21]$, among others. Recently the interest in multi-component BECs has been renewed by more detailed and more controlled experimental results illustrating the effects of phase separation [22, 23, 24], which have, in turn, motivated further theoretical studies in the subject [25, 26]; see also [27] for a recent review.

Although in the present work, we will focus on twocomponent condensates, it is relevant to note in passing the increasingly growing interest in three-component, socalled, spinor condensates [28]. Among the numerous themes of investigation within the latter context we mention spin domains [29], polarized states 30], spin textures 31], as well as multi-component (vectorial) solitons of bright 32, 33, 34, 35], dark [36], gap [37], and brightdark [38] types.

Our aim in the present manuscript is to revisit the theme of binary condensates in quasi-one-dimensional BEC settings, in an attempt to offer additional both analytical and numerical insights on the phenomenology of phase separation. Our manuscript is organized as follows. In Sec. II a Gaussian trial function is used in a variational approach to obtain six first-order ordinary differential equations (ODEs) for the time evolution of the parameters of the two-component ansatz: position, amplitude, width, phase, wave number and chirp. In Sec. III A the fixed points of the system of ODEs are obtained to yield the equilibrium position, amplitude, and width of the two species. Bifurcation diagrams of the ansatz' parameters are produced as a function of the interspecies coupling strength. In Sec. IIIB, phase diagrams are produced and analytical conditions for miscibility are obtained relating the interspecies coupling with the system's chemical potential and parabolic trap strength. The dynamics of this system is compared to 
results obtained by numerical integration of the GrossPitaevskii equation in Sec. IIIC, In Sec. IIID the system of ODEs is further reduced, upon suitable approximations, to a classical Newtonian system; the latter is simple to analyze and instructive with respect to the interpretation of the fundamental interactions driving the system's dynamics. In Sec. IV] we numerically analyze in a systematic way the existence and stability of higher excited phase-separated states as a function of the interspecies interaction. The dynamical instability evolution of the latter class of states is examined numerically in Sec. V. Motivated by the numerical experiments of Sec. V] in Sec. VI we study the existence of asymmetric states when the chemical potentials of the two components differ. Finally, in Sec. VII we summarize our results and present some interesting directions for future investigation.

\section{VARIATIONAL MODEL}

\section{A. Coupled equations}

The Gross-Pitaevskii (GP) equation, which is a variant of the NLS equation accounting for the potential confining the atomic species, governs the dynamics of bosonic particles near absolute zero temperatures [1, 2]. In the context considered herein (related to the case of ${ }^{87} \mathrm{Rb}$ which is common in relevant experiments [17, 23]), the two hyperfine states of the same atom are described by a set of coupled GP equations [9]

$$
\begin{aligned}
i \hbar \frac{\partial \psi_{1}}{\partial t} & =\left(-\frac{\hbar^{2}}{2 m} \nabla^{2}+V_{1}+g_{11}\left|\psi_{1}\right|^{2}+g_{12}\left|\psi_{2}\right|^{2}\right) \psi_{1} \\
i \hbar \frac{\partial \psi_{2}}{\partial t} & =\left(-\frac{\hbar^{2}}{2 m} \nabla^{2}+V_{2}+g_{22}\left|\psi_{2}\right|^{2}+g_{21}\left|\psi_{1}\right|^{2}\right) \psi_{2} \\
V_{j} & =\frac{1}{2}\left(\omega_{j x}^{2} x^{2}+\omega_{j y}^{2} y^{2}+\omega_{j z}^{2} z^{2}\right), j=1,2
\end{aligned}
$$

Here, $g_{j k}=4 \pi \hbar^{2} a_{j k} / m$ are the self coupling interaction parameters of the first species for $j=1, k=1$, for the second species $j=2, k=2$ and for $j=1, k=2$ or $j=2$, $k=1$ are the cross species coupling parameters. These interaction strengths depend on the scattering lengths between same $\left(a_{11}\right.$ and $\left.a_{22}\right)$ and different species $\left(a_{12}=\right.$ $\left.a_{21}\right)$. The external harmonic trapping potential for each atomic species, $V_{j}=V_{j}(\mathbf{r})$ depends on the radial distance $\mathbf{r}$ from the center of the trap, while the atomic density is given by the square modulus of $\psi_{j}=\psi_{j}(\mathbf{r}, t)$. The atomic mass is denoted by $m$.

We will make the customary assumption that the external trap's effect on each species is the same, making $V_{1}(\mathbf{r})=V_{2}(\mathbf{r})$. More importantly, in the interest of analytical tractability of our results, we will also assume that the self interactions for each species is the same, $g_{11}=g_{22}$. Both assumptions are very good approximations of the physical reality, although they are not exact; see e.g., the relevant discussion of [23].
In a highly anisotropic trap, where the frequency of the longitudinal component of the trap is much smaller than the transverse components $\omega_{x} \ll \omega_{y}=\omega_{z}$, an effective one-dimensional (1D) system of partial differential equations (PDEs) can be obtained [3]

$$
\begin{aligned}
& i \frac{\partial u_{1}}{\partial t}=\left(-\frac{1}{2} \frac{\partial^{2}}{\partial x^{2}}+\frac{\Omega^{2}}{2} x^{2}+\left|u_{1}\right|^{2}+g\left|u_{2}\right|^{2}\right) u_{1}, \\
& i \frac{\partial u_{2}}{\partial t}=\left(-\frac{1}{2} \frac{\partial^{2}}{\partial x^{2}}+\frac{\Omega^{2}}{2} x^{2}+\left|u_{2}\right|^{2}+g\left|u_{1}\right|^{2}\right) u_{2},
\end{aligned}
$$

where time, space, and wave function have been rescaled to reduce the system's parameters to just two $(\Omega$ and $g)$. In this $1 \mathrm{D}$ reduction, the chemical potential corresponds to $\mu_{1 D}, \psi_{j} \rightarrow u_{j} \sqrt{g_{j j} / \mu_{1 D}}, x \rightarrow x \sqrt{m \mu_{1 D}} / \hbar$ is the longitudinal distance from the center of the trap, $t \rightarrow t \mu_{1 D} / \hbar$ is the rescaled time, $g=g_{12} / g_{11}=g_{21} / g_{22}$ is the rescaled species interaction term. In the literature, the condition of miscibility $\Delta=\left(g_{12} g_{21}-g_{11} g_{22}\right) / g_{11}=g^{2}-1$ is often used see e.g. [8, [9, 10]. The rescaled harmonic trap frequency is given by $\Omega=\hbar \omega_{x} / \mu_{1 D}$.

\section{B. Ansatz and Euler-Lagrange Equations}

To develop a variational model, we substitute in the rescaled two-component Lagrangian

$$
\mathcal{L}=\int_{-\infty}^{\infty}\left(L_{1}+L_{2}+L_{12}+L_{21}\right) d x
$$

where

$$
\begin{aligned}
L_{j} & =E_{j}+\frac{i}{2}\left(u_{j} \frac{\partial u_{j}^{*}}{\partial t}-u_{j}^{*} \frac{\partial u_{j}}{\partial t}\right), \\
E_{j} & =\frac{1}{2}\left|\frac{\partial u_{j}}{\partial x}\right|^{2}+V(x)\left|u_{j}\right|^{2}+\frac{1}{2}\left|u_{j}\right|^{4}, \\
L_{12} & =L_{21}=\frac{1}{2} g\left|u_{1}\right|^{2}\left|u_{2}\right|^{2},
\end{aligned}
$$

the Gaussian ansatz of the form

$$
\begin{aligned}
& u_{1}(x, t)=A e^{-\frac{(x-B)^{2}}{2 W^{2}}} e^{i\left(C+D x+E x^{2}\right)}, \\
& u_{2}(x, t)=A e^{-\frac{(x+B)^{2}}{2 W^{2}}} e^{i\left(C-D x+E x^{2}\right)} .
\end{aligned}
$$

Starred variables indicate complex conjugation. The parameters $A, B, C, D, E$, and $W$ are assumed to be time dependent and they represent the amplitude, position, phase, wave number, chirp, and width of the Gaussian ansatz, respectively. For large $\Omega$, the steady state solution very closely resembles two Gaussian functions separated by a distance $2 B$, with constant rotation of the phase $C=\mu t$, where $\mu$ is the chemical potential (without loss of generality we take $\mu=1$ ). Upon interaction between the species and with the harmonic trap, acceleration induces an inhomogeneity in the carrier wave, known as chirp, that accordingly affects the phase of each 
species. It is important to note here that the ansatz is invariant upon transposition of space and atomic species component $x \rightarrow-x$ and $u_{1} \rightarrow u_{2}$. This allows us to reduce the number of parameters in the system to six instead of having twelve, which would take into account independent variation of the parameters in $u_{1}$ and $u_{2}$. However, this simplification comes at a certain cost as, in particular, it is not possible to monitor asymmetric (between the two components) states within this ansatz; the latter type of states will be partially explored within the dynamics of the species in Sec. $\mathrm{V}$.

When the Lagrangian is evaluated for the proposed ansatz, a spatially-averaged effective Lagrangian is obtained

$$
\begin{aligned}
\mathcal{L}= & -\sqrt{\pi} A^{2} W\left[\Omega^{2} B^{2}+2 E^{2} W^{2}+(D+2 B E)^{2}\right. \\
& +\frac{A^{2}}{\sqrt{2}}\left(1+g e^{-\frac{2 B^{2}}{W^{2}}}\right)+\frac{1}{2 W^{2}}+\frac{\Omega^{2} W^{2}}{2} \\
& \left.+2 \frac{d C}{d t}+2 B \frac{d D}{d t}+\frac{d E}{d t}\left(2 B^{2}+W^{2}\right)\right]
\end{aligned}
$$

and the equations of motion for the parameters are obtained through the corresponding Euler-Lagrange equations

$$
\frac{\partial L}{\partial p_{j}}-\frac{d}{d t}\left(\frac{\partial L}{\partial \dot{p_{j}}}\right)=0
$$

where the parameter $p_{j}, j=1,2, \ldots, 6$ represents the parameters in the ansatz $A, B, C, D, E, W$ and $\dot{p}_{j}=$ $d p_{j} / d t$. The Euler-Lagrange equations for $A, B$, and $W$ evaluate to $\partial L / \partial A=0, \partial L / \partial B=0$ and $\partial L / \partial W=0$ since the second term in the Euler-Lagrange equation (77) is zero for these. This results in equations that involve linear combinations of $d C / d t, d D / d t$ and $d E / d t$ that when solved, give Eqs. (10), (11) and (12). The rest of the Euler-Lagrange equations give equations that involve linear combinations of $d A / d t, d B / d t$ and $d W / d t$ that can be solved to give Eqs. (8), (91) and (13). The following equations are the result of solving the EulerLagrange equations for the time derivatives of all the pa- rameters of our ansatz:

$$
\begin{aligned}
& \frac{d A}{d t}=-A E, \\
& \frac{d B}{d t}=D+2 B E, \\
& \frac{d C}{d t}=\frac{B^{2}}{2 W^{2}}-\frac{D^{2}}{2}-\frac{1}{2 W^{2}}+\frac{\sqrt{2} A^{2}}{8 W^{2}}\left(2 B^{2}-5 W^{2}\right)+ \\
& \frac{\sqrt{2} A^{2} g}{8 W^{2}} e^{-\frac{B^{2}}{2 W^{2}}}\left(8 B^{4}+2 B^{2} W^{2}+5 W^{4}\right), \\
& \frac{d D}{d t}=\frac{\sqrt{2} A^{2} B g}{2 W^{4}} e^{-\frac{B^{2}}{2 W^{2}}}\left(4 B^{2}+W^{2}\right)- \\
& \frac{\sqrt{2} A^{2} b}{2 W^{2}}-\frac{B}{W^{4}}-2 D E, \\
& \frac{d E}{d t}=\frac{\sqrt{2} A^{2} g}{4 W^{4}} e^{-\frac{B^{2}}{2 W^{2}}}\left(-4 B^{2}+W^{2}\right)+ \\
& \frac{\sqrt{2} A^{2}}{4 W^{2}}+\frac{1}{2 W^{4}}-2 E^{2}-\frac{\Omega^{2}}{2}, \\
& \frac{d W}{d t}=2 E W \text {. }
\end{aligned}
$$

As described below, these ODEs reflect fairly accurately the principal dynamical features of the system. In particular, they capture the oscillations of the two species past each other when the equilibrium separation $B$ is zero and the oscillations about their corresponding phase-separated equilibrium position when the two components are phase-separated.

\section{STEADY STATE SOLUTIONS}

\section{A. Phase Bifurcations}

The steady state of Eqs. (8) -(13) is obtained by setting $d A / d t=d B / d t=d C / d t=d E / d t=d D / d t=d E / d t=$ $d W / d t=0$ witch leads immediately to the steady state solution $E_{*}=D_{*}=0$ and $C_{*}=\mu$. When the equilibrium separation between the species is zero, the equilibrium amplitude and width reduce to

$$
\begin{aligned}
B_{*} & =0, \\
A_{*}^{2} & =\frac{2 \sqrt{2}\left(8 \mu-\sqrt{15 \Omega^{2}+4 \mu^{2}}\right)}{15(1+g)}, \\
W_{*}^{2} & =\frac{\left(2 \mu+\sqrt{15 \Omega^{2}+4 \mu^{2}}\right)}{5 \Omega^{2}} .
\end{aligned}
$$

When the equilibrium separation is nonzero, the resulting equilibrium amplitude and width are given by the 

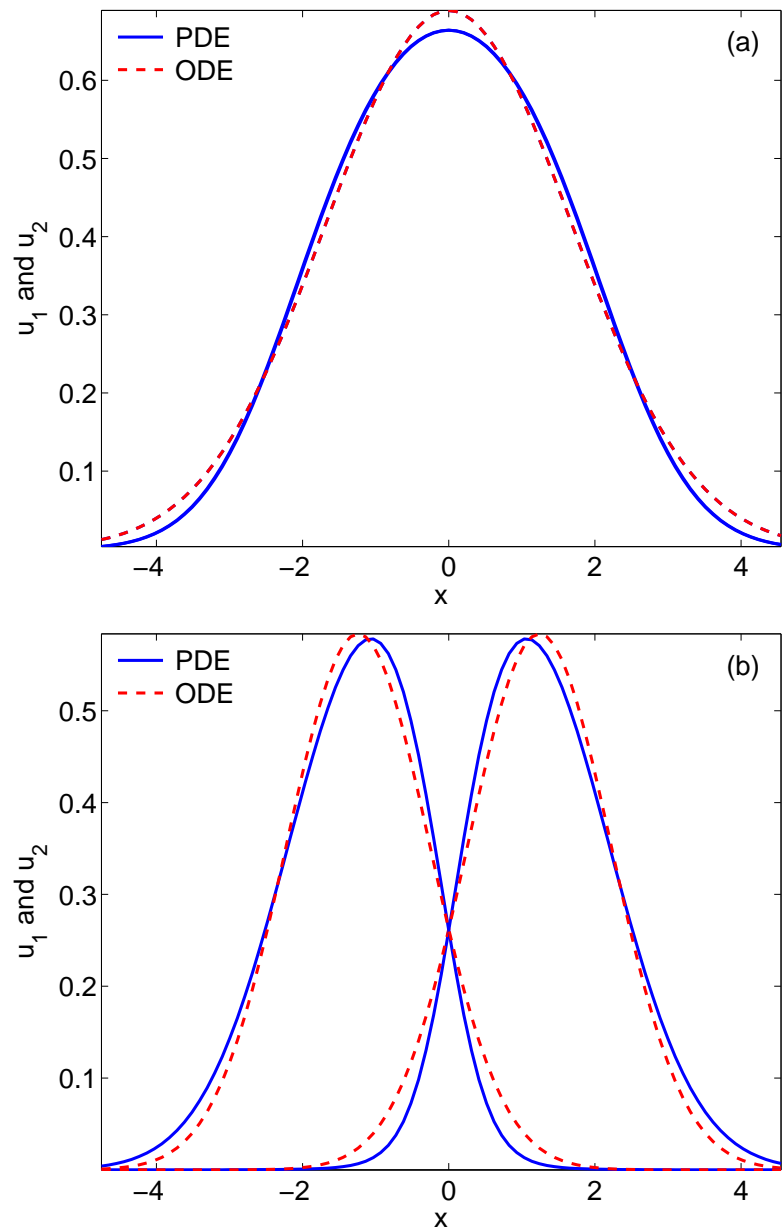

FIG. 1: (Color online) (a) Steady state solution, $u_{1}$ and $u_{2}$, for the mixed state when $B=0$. (b) Steady state solution for the separated state when $B \neq 0$. The solid (blue) line represents the steady state of the GP equations and the dashed (red) line is the steady state solution of the system of ODEs. Here, $\Omega=0.6, \mu=1 g=1$ for (a) and $g=20$ for (b).

transcendental relations

$$
\begin{aligned}
\Omega^{2}-\frac{\sqrt{2} A_{*}^{2} g}{W_{*}^{2}} e^{-\frac{B_{*}^{2}}{2 W_{*}^{2}}} & =0, \\
\mu-\frac{1}{2 W_{*}^{2}}-\frac{5 W_{*}^{2}}{8}\left(\sqrt{2} A_{*}^{2} g+\Omega^{2} W_{*}^{2}\right) & =0, \\
\mu+\frac{3}{4 W_{*}^{2}}-\frac{5}{4} \Omega^{2}\left(W_{*}^{2}+2 B_{*}^{2}\right) & =0 .
\end{aligned}
$$

Figure 1 shows that the steady state solution of the full GP model closely matches the steady state from the ODEs, indicating that the ansatz successfully captures the relevant PDE behavior. For large values of $\Omega$, the steady state solution of the GP deviates from the Gaussian shape, resembling an inverted parabola, often referred to as the Thomas-Fermi approximation [1, 2].

Detailed bifurcation diagrams for the amplitude, position, and width of each species can be obtained by
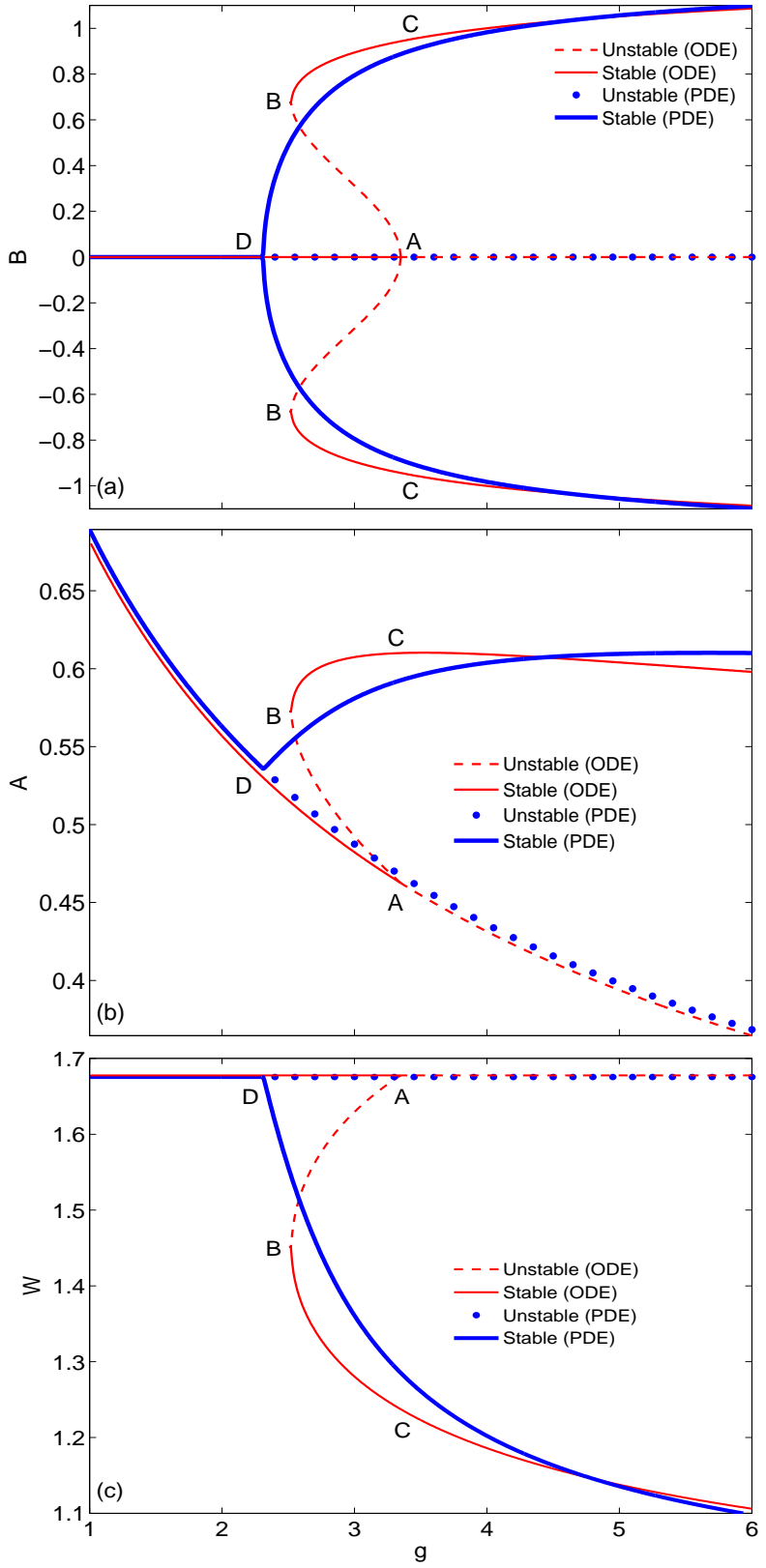

FIG. 2: (Color online) Equilibrium (a) separation, (b) amplitude, and (c) width for the two condensed species, as a function of the interspecies coupling strength $g$ for $\Omega=0.6$ and $\mu=1$. The (red) thin solid (stable) and dashed (unstable) lines correspond to the steady states for the reduced ODE model [Eqs. (8)-(13)], while the (blue) thick solid (stable) and dots (unstable) correspond to the steady state from the full GP model [Eqs. (2)-(3)].

solving Eqs. (17)-(19) for $A, B$, and $W$ for the phase separated state and Eqs. (14)-(16) for the mixed state. The steady state solution reveals a pitchfork bifurcation for the position of each condensate as the interspecies coupling strength is increased as can be seen in Fig. 23. Interestingly, the steady state of the GP equations produces a supercritical pitchfork bifurcation at point D of 
Fig. 2. More specifically, for small values of $g$, a stable mixed phase can be identified; as $g$ is increased, the mixed phase becomes unstable and the phase separated state becomes stable. On the other hand, the system of ODEs also predicts a pitchfork bifurcation, however the approximate nature of the ansatz results in the identification of the bifurcation as a subcritical one (point A) occurring in the vicinity of a symmetric pair of saddle node bifurcations (points B). Both bifurcation diagrams agree with each other away from the transition between phases. In the vicinity of the transition point, clearly, the nature of the ansatz is insufficient to capture the fine details of the bifurcation structure (thus inaccurately suggesting a phenomenology involving bistability, and hysteretic behavior of the system). It should be noted that the phase separation from the GP model (point D) lies near the saddle node bifurcation point (point B) and the subcritical bifurcation point (point A) from the system of ODEs. At small values of the harmonic trap strength, the true point of phase transition is closer to the subcritical bifurcation point and at larger values of the harmonic trap strength, the true point of phase transition lies closer to the saddle node bifurcation point.

\section{B. Phase Separation}

Using the zero separation amplitude and width, point A in Fig. 2, an expression for the onset of phase separation can be approximated in terms of the critical interspecies interaction

$$
g_{\mathrm{cr}}=\frac{6 \mu+3 \sqrt{15 \Omega^{2}+4 \mu^{2}}}{26 \mu-7 \sqrt{15 \Omega^{2}+4 \mu^{2}}} .
$$

Despite the deviation of point A in Fig. 2, from the relevant point $\mathrm{D}$ of the corresponding $\mathrm{PDE}$, the analytical expression offers valuable insight on the dependence of the critical interspecies interaction for phase separation on parameters of the trap (in particular, its frequency) and those of the condensate (in particular, its chemical potential); see also Fig. 3 for a detailed comparison of the ODE and PDE bifurcation points. More specifically, the equation predicts that when the harmonic trap's frequency approaches zero, $\Omega \rightarrow 0$, phase separation occurs when $g_{\text {cr }} \rightarrow 1$ coinciding with the well known miscibility condition $\Delta=g^{2}-1=0$. As $\Omega \rightarrow \Omega_{\mathrm{cr}}=4 \sqrt{2} \mu / 7 \approx 0.8 \mu$ phase separation occurs at $g_{\text {cr }} \rightarrow \infty$. This behavior can be qualitatively understood since tighter (larger $\Omega$ ) traps tend to "squeeze" both components together, thus frustrating the system's tendency towards phase separation.

The prediction of the system of ODEs for the location of the bifurcation point agrees well with the results from the GP model for small values of the harmonic trap strength. Recall that the phase separation for the ODE model is located at the subcritical pitchfork bifurcation point A in Fig. 2. However, as can be observed from

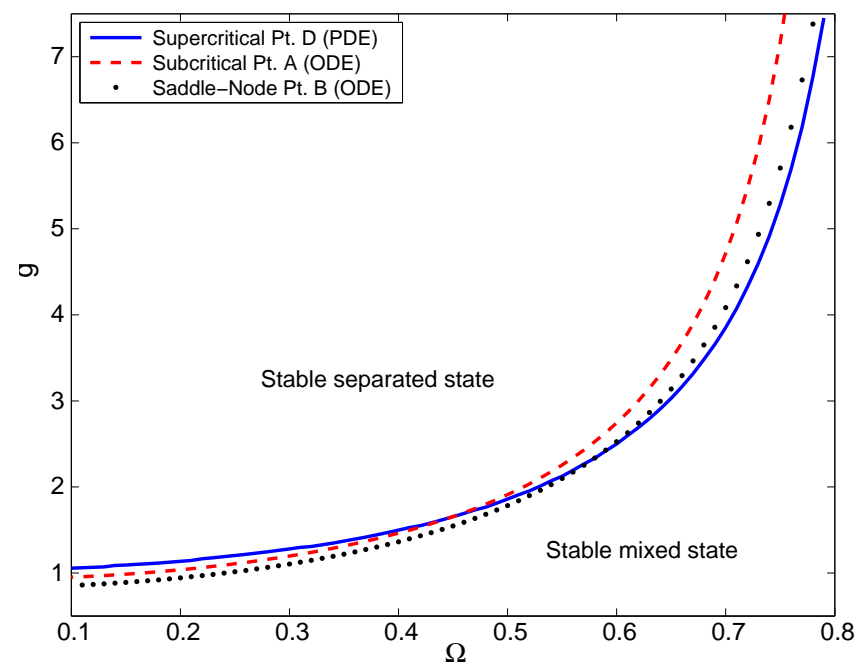

FIG. 3: (Color online) The (blue) solid line represents the boundary of zero species separation from the GP equations (point D in Fig. 2), where states that lie to the right of this line are mixed and values to the left are phase-separated. The (red) dashed line represents the boundary of zero species separation for the system of ODEs given by Eq. (20) (point A in Fig. 2). The (black) dotted line shows the saddle-node point (point B in Fig. 2) obtained from the system of ODEs.

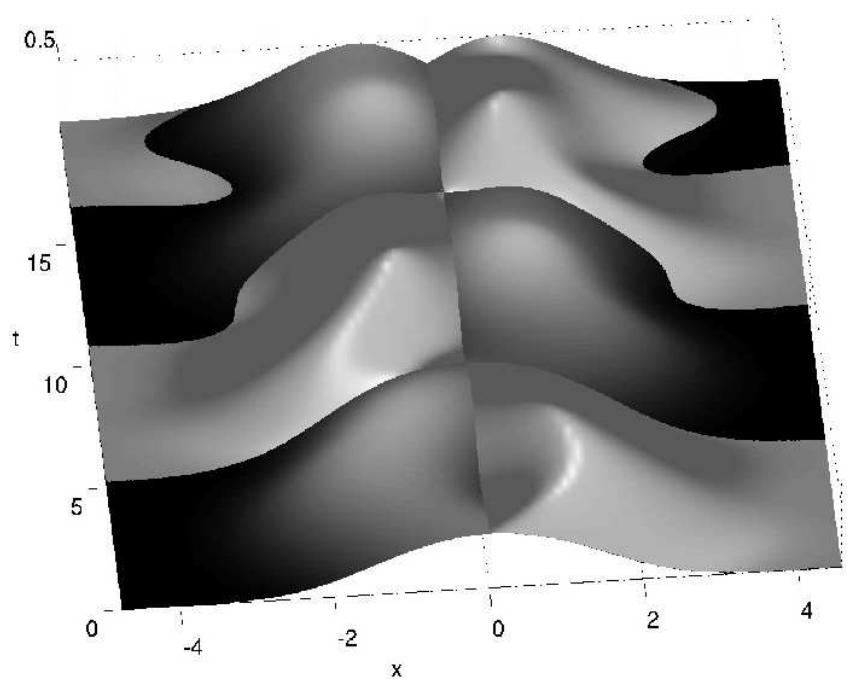

FIG. 4: Oscillations for a mixed state from direct numerical integration of the GP model (2)-(3) for $g=1\left(g<g_{\mathrm{cr}}\right), \mu=1$ and $\Omega=0.6$. Lighter gray corresponds to one the density of one component and darker gray to the other component.

Fig. 3, a better approximation for the full system's phase separation (point D), in the case of large trapping frequencies, can be given by the saddle-node bifurcation point B for the ODE reduced system for $\Omega$ values close to $\Omega_{\mathrm{cr}}$. 

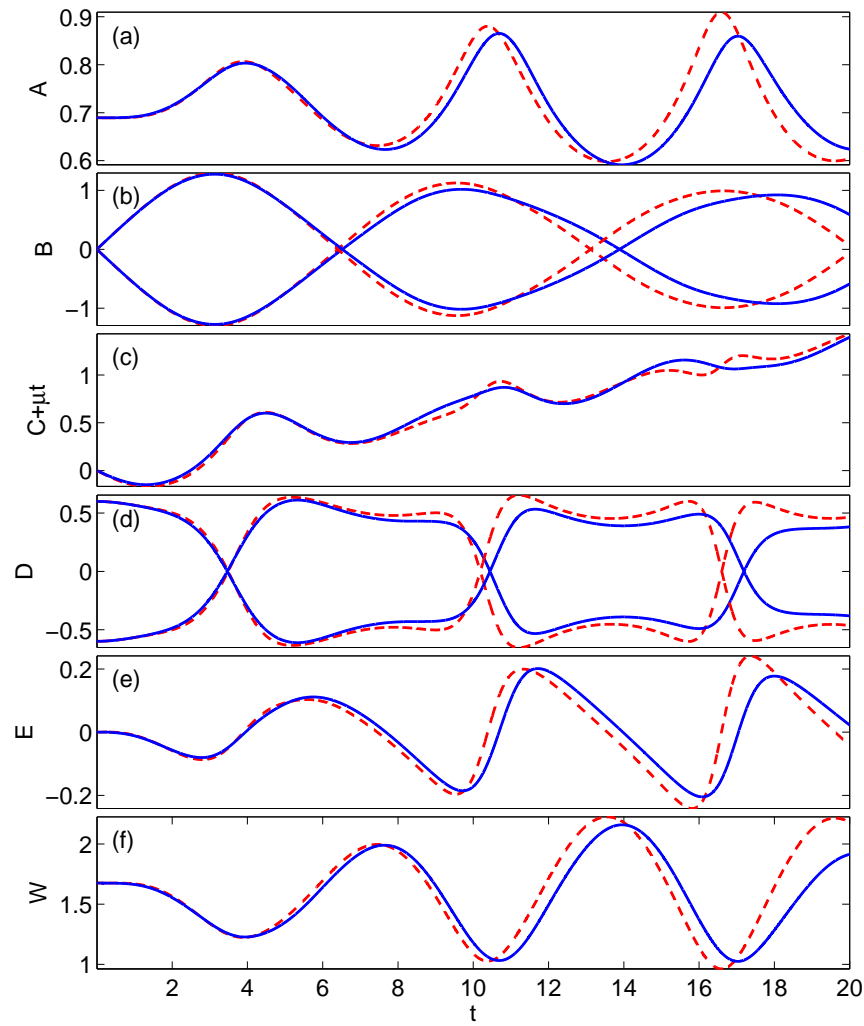

FIG. 5: (Color online) (a) Amplitude, (b) position, (c) phase, (d) velocity, (e) chirp, and (f) width corresponding to the mixed oscillating state show in Fig. 4. Solid lines represent results from direct numerical simulations of the GP equation while dashed lines depict the results for the ODE reduction (9) - (13)

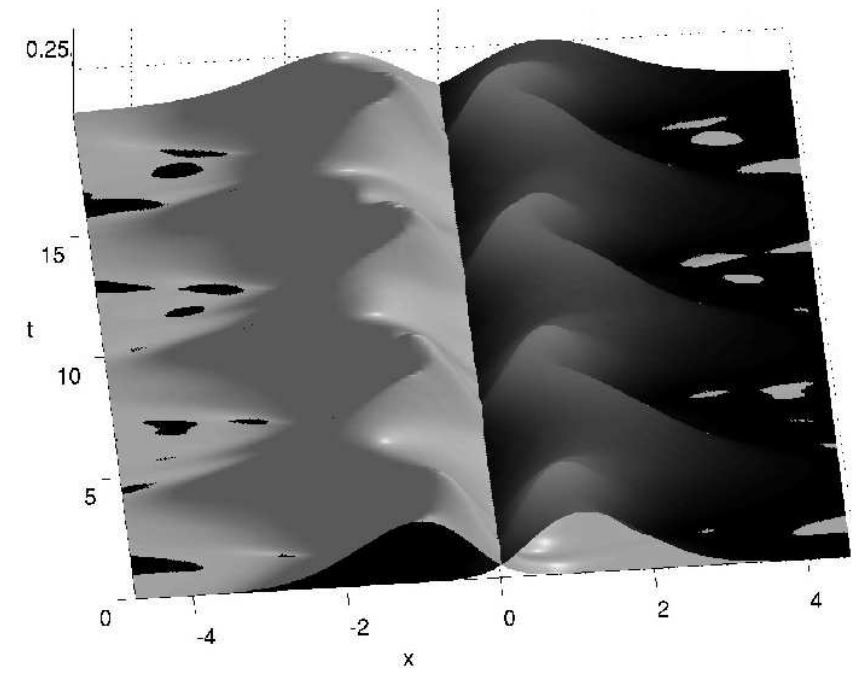

FIG. 6: Same as in Fig. 4 for the time evolution of oscillating species about their phase separated configuration when $g=$ $20\left(g>g_{\mathrm{cr}}\right)$.

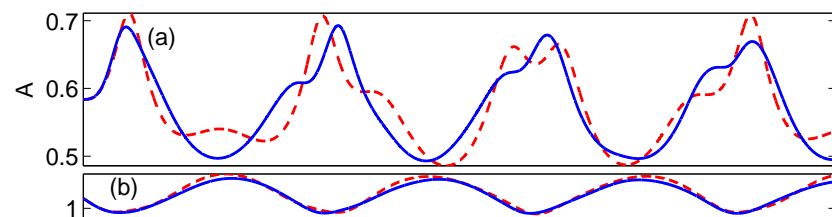

$\infty \quad 0$
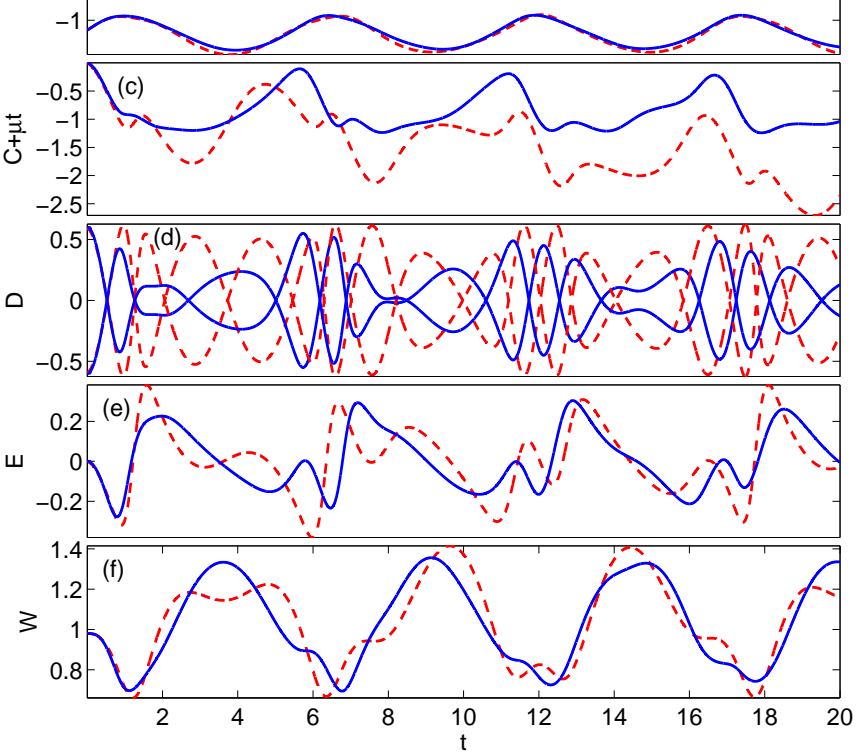

FIG. 7: (Color online) Same as in Fig. 5 for the time evolution of oscillating species about their phase separated configuration as shown in Fig. 6]

\section{Dynamics of the Reduced System}

For relatively small values of the interspecies coupling $g$ (i.e., to the left of point D in Fig. 2) the two species do not separate and thus, when given opposite direction velocities from the mixed state, they will oscillate through each other as depicted in Fig. 4. This case is analyzed in Fig. [5 where the two components oscillate through each other about their common equilibrium separation of zero and the prediction from the system of ODEs (red dashed lines) agrees very well with the direct integration of the GP equations (blue solid lines). Because of conservation of mass, the amplitude and width oscillate out of phase: the amplitude is maximized and width is minimized when the acceleration of the species is maximized; on the other hand, the width is maximized and the amplitude is minimized when the velocity is maximized. The velocity of each species [see Eq. (9)] has two components, one that depends on the wave number $D$ and another that depends on the product of chirp and position $2 E B$. If there is no chirp, the wave number is the velocity of the condensate species. In Fig. 5F and 7 r, a factor of $\mu t$ has been added to show the deviation of the phase from the steady state value.

On the other hand, if we assume a well-separated state as the PDEs initial condition, (right of point D in Fig. 2), it is possible that the two components entertain oscil- 


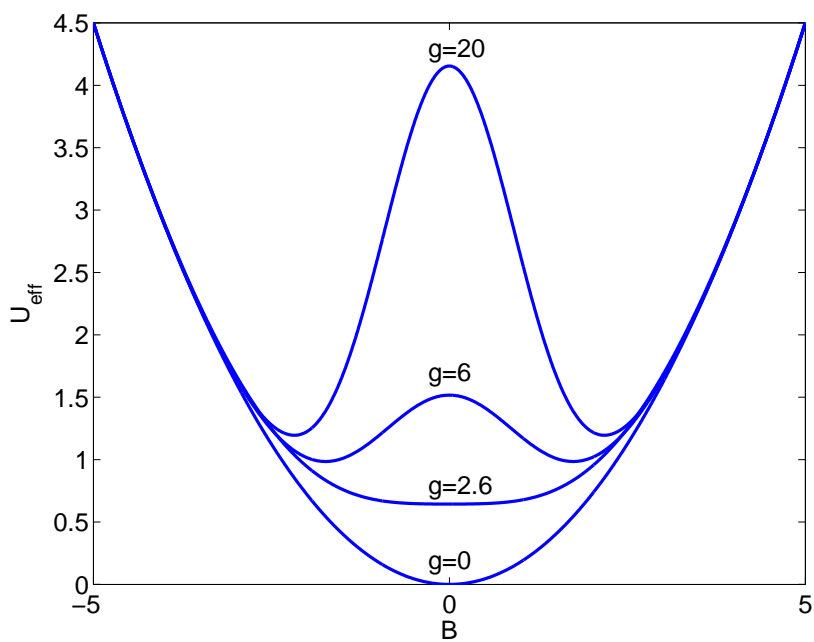

FIG. 8: (Color online) The effective potential, $U_{\text {eff }}$, for several values of the species coupling parameter: $g=0, g=2.6$, $g=6$, and $g=20$. Here, $\Omega=0.6$ and $\mu=1$.

lations about their phase-separated steady state as depicted in Fig. 6. As illustrated in Fig. 7 the two phase separated components collide against each other as they oscillate (but do not go through each other) about a nonzero position. The prediction from the system of ordinary differential equations once again agrees very well with the numerical integration of the GP equation for the position of the two species, but differs somewhat for the other parameters of motion. This occurs because the time evolution of the solution of Eqs. (2) and (3) in this case, due to the oscillation and interaction, deviates from a Gaussian waveform and the corresponding variational prediction begins to lose accuracy.

\section{Newtonian Reduction}

To develop a more tractable model for the dynamics, a classical Newtonian system for the motion of the center of each species is desirable. Taking a time derivative of Eq. (9) and substitution of Eqs. (9), (11), and (12) yields

$$
\frac{d^{2} B}{d t^{2}}=-\left(\Omega^{2}-\frac{\sqrt{2} A_{*}^{2} g}{W_{*}^{2}} e^{-\frac{B^{2}}{2 W_{*}^{2}}}\right) B,
$$

where we simplified the dynamics by replacing the time dependent variations of $A$ and $W$ by their respective equilibrium values $A_{*}$ and $W_{*}$. This simplification is justified by the fact that the oscillations in $A$ and $W$ are relatively weak as it can be observed from Figs. 5a, 5 5 , 7a, and 7f. These phase separated oscillations contain two fundamental physical features: the external trapping potential and an exponential repulsive interaction that depends on the cross species coupling parameter $g$. Integrating Eq. (21) with respect to $B$ yields a Newtonian equation
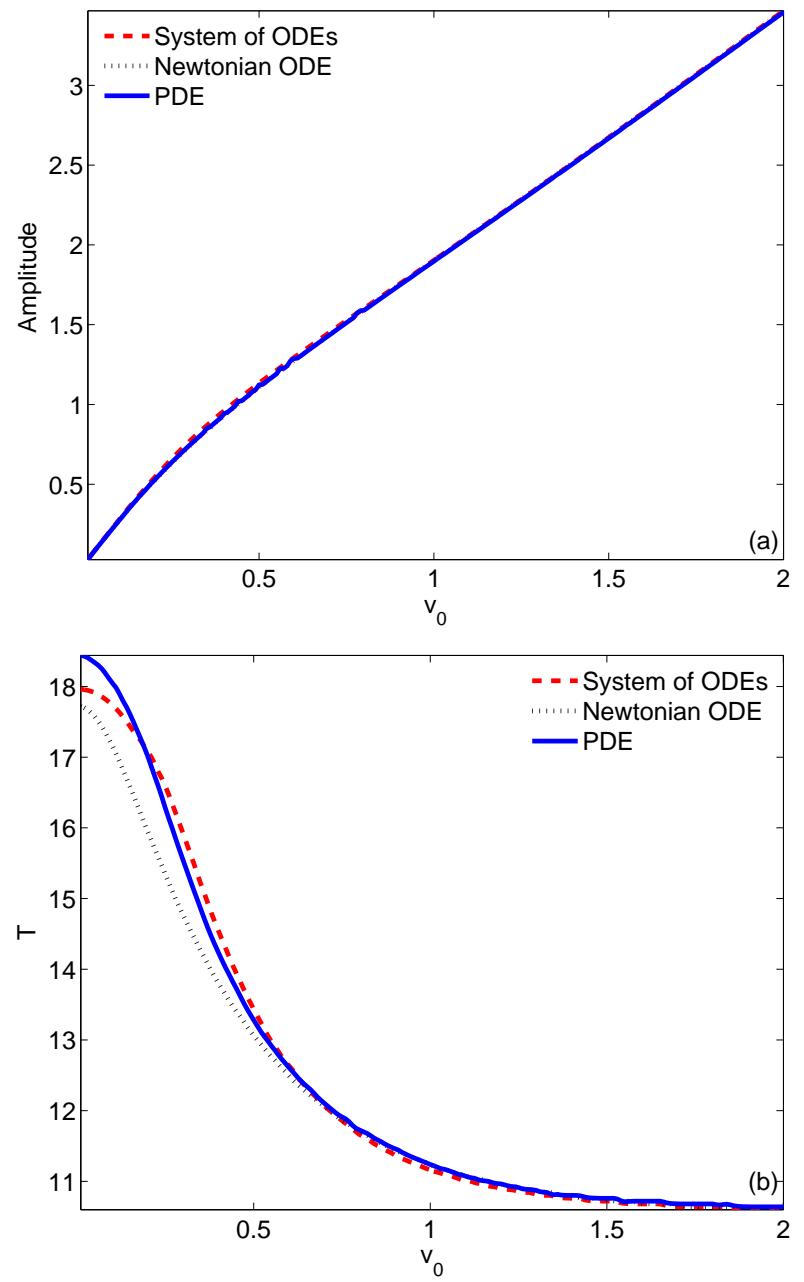

FIG. 9: (Color online) (a) Amplitude of oscillation and (b) period of oscillation, $T$, as a function of increasing initial velocity, $v_{0}$. The (blue) solid line represents results from the coupled GP equations, the (red) dashed line represents results from the system of ODEs, and the black dotted line represents the results from the Newtonian reduction. Here, $g=1, \mu=1$ and $\Omega=0.6$.

of motion under the effective potential

$$
U_{\text {eff }}=\frac{\Omega^{2}}{2} B^{2}+\frac{\sqrt{2} A_{*}^{2} g}{2} e^{-\frac{B^{2}}{2 W_{*}^{2}}} .
$$

This reduced dynamics gives an effective double well potential for a fully phase separated state $\left(g>g_{\text {cr }}\right)$ and a nonlinear single well potential for the mixed states $\left(g<g_{\text {cr }}\right)$. It is important to note that in the expression of Eq. (22), $A_{*}$ and $B_{*}$ vary as a function of $g$. This dependence has been incorporated in Fig. 8 which shows the effective potential for increasing values of $g$, where it transitions from a single well potential to a double well potential. For $\Omega=0.6$ and $\mu=1$, the reduced Newtonian model predicts that at $g=2.6$ the two species phase separate and the potential becomes very flat yielding very long periods of oscillation. It is remarkable to point out that a similar picture has been drawn for the interaction 

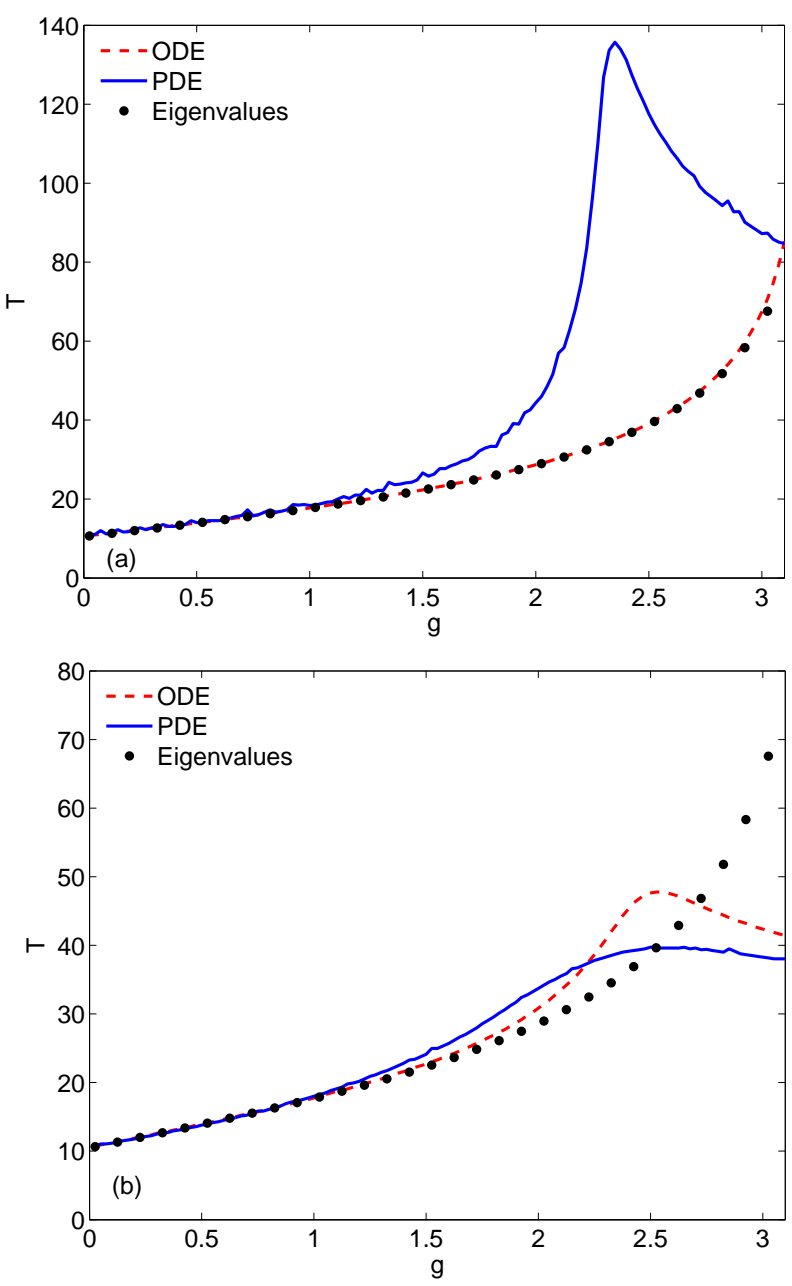

FIG. 10: (Color online) (a) The period of oscillation, $T$, is shown as a function of $g$ for an initial velocity of $v_{0}=0.01$. (b) The period of oscillation, as a function of $g$ for an initial velocity of $v_{0}=0.1$. The (blue) solid line represents results from the coupled GP equations, the (red) dashed line represents results from system of ODEs, while the black dots represent the results from the evaluation of the eigenvalues of the Jacobian of the system of ODEs at equilibrium. Here, $\Omega=0.6$ and $\mu=1$.

of two particle-like excited states (i.e., dark solitary matter waves) within the same species and has been found to be extremely successful in comparisons with experimental results [39]. In that case, as well, the fundamental characteristics were the parabolic trapping and the exponential tail-tail interaction between the waves.

Figure 9 depicts both the (a) amplitude and (b) period of oscillations predicted by the Newtonian reduction in comparison with the corresponding PDE findings. It is seen that the GP equation and the ODE system agree very well for a wide range of values of initial velocities. This figure also shows the nonlinearity of oscillations where small amplitude oscillations have a period of 18.3 and large oscillations yield a period of $T \approx 10.5$, which corresponds to the harmonic trap's period $T \rightarrow 2 \pi / \Omega$.
Figure 10a shows that the period of oscillation from the system of ODEs matches that of the GP model for small values of $g$. As $g \rightarrow 2.4$ (i.e., when approaching phase separation of the GP equations [point D in Fig. 2]), the period changes substantially. Then as $g \rightarrow 3.1$, the system of ODEs begins to phase-separate. Since these are small oscillations, the eigenvalues from the Jacobian of the system of ODEs at equilibrium matches very well the oscillations of the system of ODEs. Figure10 b shows similar results to Fig. 10 but for larger oscillations. We can see that for larger oscillations the ODEs' period more closely matches that of the GP for smaller $g$, while for larger values of the interspecies strength, the deviation becomes more significant. Furthermore, for larger values of $g$, the period obtained from the eigenvalues also deviates from the period from the ODEs. For large oscillations, the two species do not effectively interact (since they go through each other too rapidly to "feel" each other) and the period is roughly independent of $g$ as predicted by Eq. (21) when $|B| \ll 1$, yielding simple harmonic oscillations. In this case, the GP's period is close to that predicted by the ODEs.

\section{EXCITED STATES}

For sufficiently weak traps $\Omega<0.5$, as the interspecies coupling strength is increased for $g>1$, new, high order mixed states emerge. These higher excited states correspond to alternating bands dominated successively by each of two species. As $\Omega$ is decreased or alternatively $g$ is increased, the number of alternating bands increases within the solution profile and the population imbalance within each band less pronounced. Each solution branch is found by using parameter continuation on the parameter $g$ using a Newton fixed point iteration to find the stationary state. The stability for each computed profile was obtained by computing the eigenvalues of the linearized dynamics at the fixed points using standard techniques [3]. We now describe the series of bifurcations that occur as the interspecies coupling $g$ increases as depicted in Fig. 11:

- For $g<1.08$, the only state that exists is the mixed state and it is stable. This threshold is close to the traditional miscibility condition $\Delta=g^{2}-1=0$.

- At $g=1.08$ (point A), the mixed states becomes unstable, through the previously described supercritical pitchfork bifurcation, leading to the emergence of a stable phase-separated state where one component moves to the left and the other one moves to the right.

- At $g=1.24$ (point B), a second supercritical pitchfork occurs, rendering the symmetric (across components) solution more unstable and giving rise to a state where one species has a single hump and the other one has a double hump. We call this state a 1-2 hump configuration. 


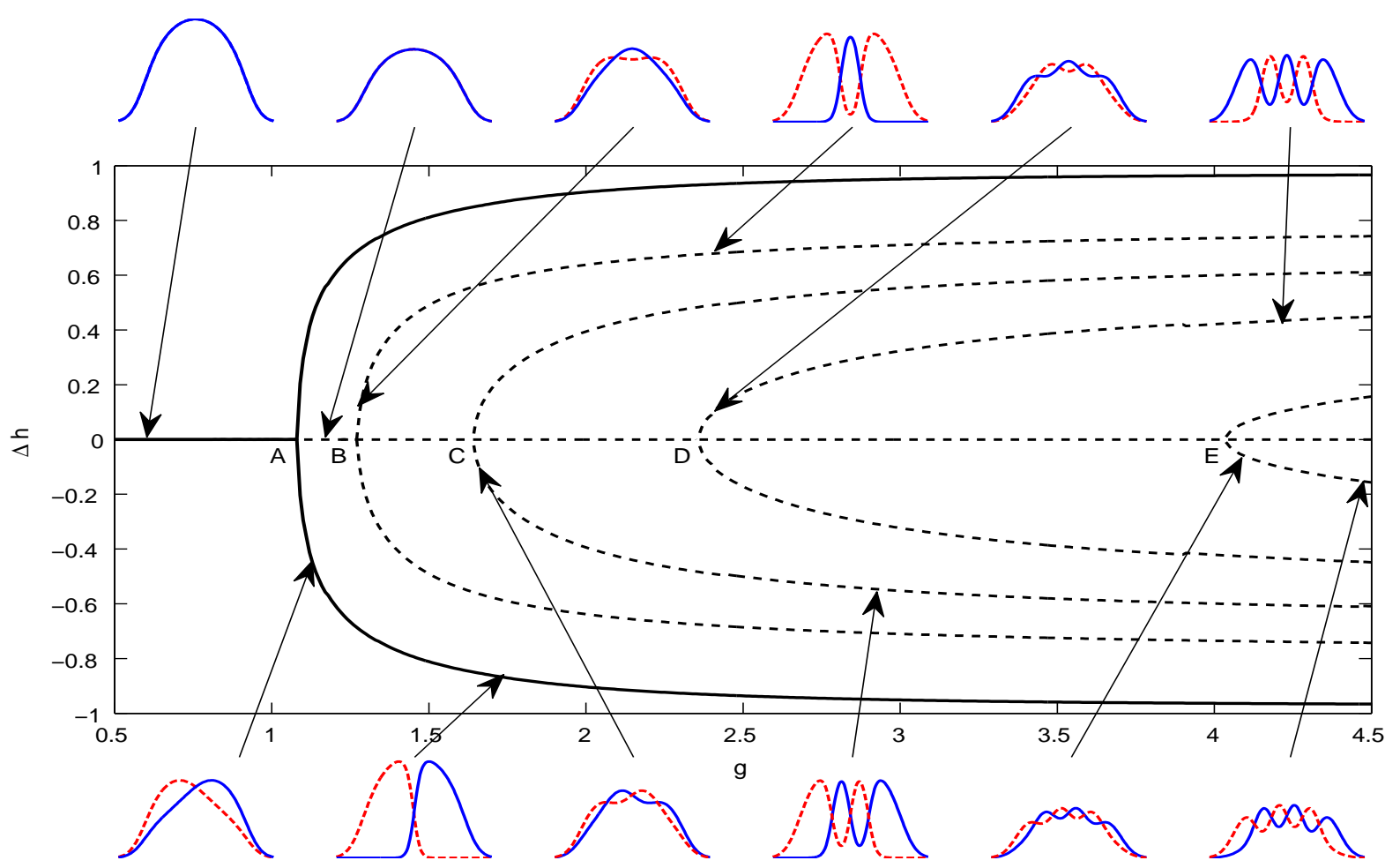

FIG. 11: (Color online) Degree of phase separation $\Delta h=u_{1}\left(x_{c}\right)-u_{2}\left(x_{c}\right)$, where $x_{c}$ is the location of the closest density maximum (irrespective of component) to the trap center, as a function of the interspecies coupling parameter for various excited states for $g \in[0.5,4.5]$ and $\Omega=0.2$. Stable (unstable) solutions branches are depicted with black solid (dashed) lines. Typical solutions for each branch are depicted in the surrounding insets.

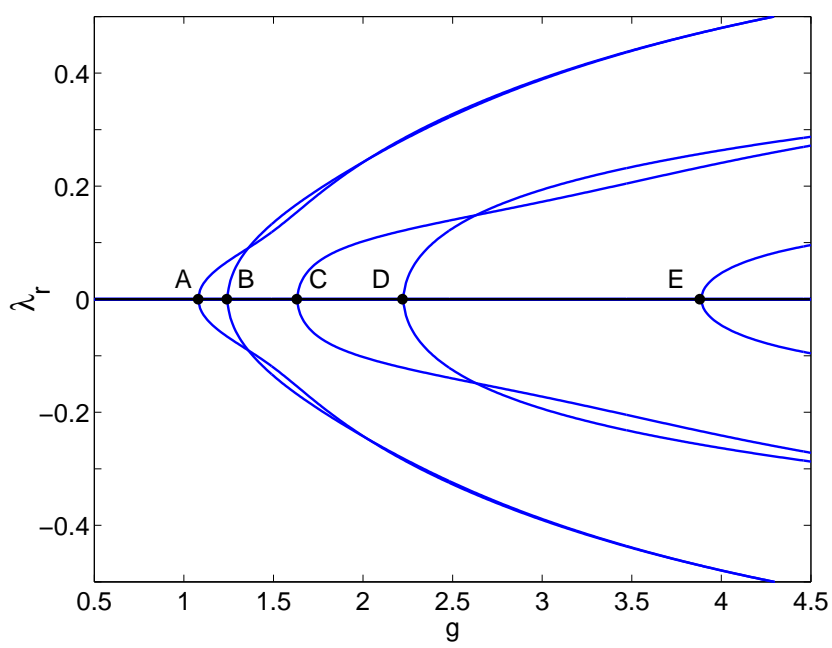

FIG. 12: (Color online) Real part of largest eigenvalue of the mixed state as a function of the interspecies coupling parameter for the various excited states depicted in Fig. 11] with $g \in[0.5,4.5]$, and $\Omega=0.2$.

- At $g=1.63$ (point C), the third supercritical pitchfork bifurcation of the series arises, leading this time to a situation where one species moves to the left and the other one moves to the right, both forming a double hump (a 2-2 hump configuration).

- At $g=2.22$ (point D), a double hump with a triple hump state arises (a 2-3 hump configuration).

- At $g=3.8$ (point E), a triple hump with a triple hump state forms (a 3-3 hump configuration).

As the condensate becomes wider, more and more bands appear, each band having a width comparable to the healing length of the condensate. This picture seems to be natural from the point of view of [25], where the emergence of these higher excited states could be interpreted as a manifestation of an effective modulational instability. Within the effectively finite region determined by the confining potential, as $g$ is increased, higher "modulation wavenumbers" become unstable, leading to the "quantized" (associated with the quantization of wavenumbers in the effectively finite box) cascade of supercritical pitchfork bifurcations and associated further destabilizations of the symmetric state. The degree of phase separation in Fig. 11 between these bands is computed as $\Delta h=u_{1}\left(x_{c}\right)-u_{2}\left(x_{c}\right)$, where $x_{c}$ is the location of the closest density maximum, irrespective of component, to the trap center.

The emergence of high order states can be inferred by observing the real part of the eigenvalues as the interspecies coupling is varied. The eigenvalues collide 
with the imaginary axis as new states emerge. Eigenvalue analysis shows that all excited states are unstable with the exception of the single-hump phase-separated state (which results from the first pitchfork bifurcation, namely the 1-1 hump state). These excited states are, however, less unstable than the mixed state from which they arise. The latter, as shown in Fig. 12, becomes progressively more unstable, as expected, as further multihump branches arise. Each of the bifurcation points, for which the same designation as in Fig. 11 is used, corresponds to a further pair of real eigenvalues appearing for the mixed branch.

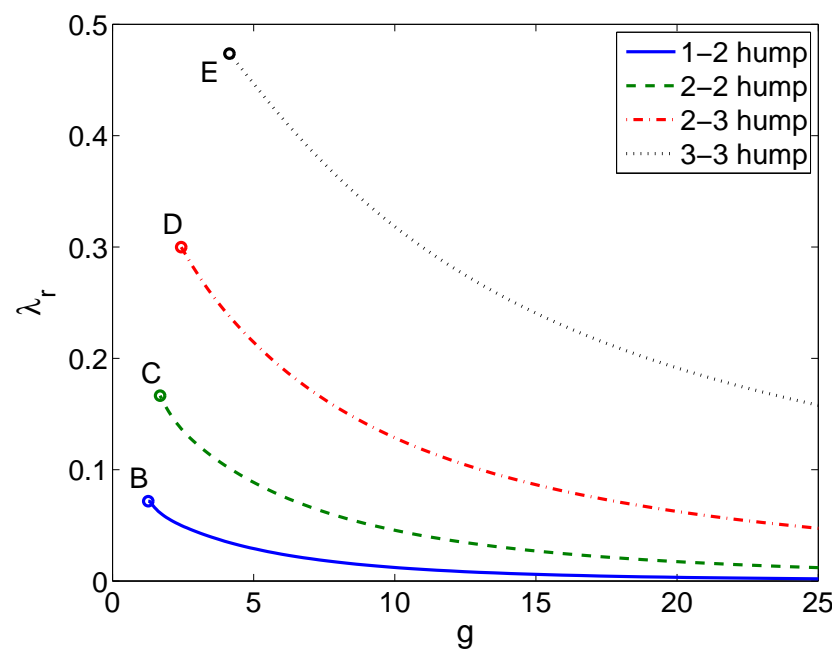

FIG. 13: (Color online) Real part of the largest eigenvalues of the principal phase-separated states as a function of the interspecies coupling parameter, $g$, for $\Omega=0.2$.

As indicated by Fig. 11, the 1-1 hump phase separated state is stable even for large values of $g$. In fact, for $\Omega \ll 1$ and $g \gg 1$, the two components repel each other so strongly that the center of magnetic trap becomes a domain wall, where each species abruptly transitions from near zero atomic density to maximum atomic density. Apparently, this 1-1 hump phase-separated state is the only stable state of the system after the mixed state loses its stability past the bifurcation point A (see Fig. 11). Nonetheless, as depicted in Fig. 13, the additional multi-humped excited states are successively created as $g$ is increased (bifurcation points B, C, D, and $\mathrm{E}$ in Fig. 11) and are progressively less unstable as $g$ increases. Therefore, for sufficiently large $g$, the instability of higher excited multi-humped states might be weak enough for these states to be observable within experimentally accessible times. It is interesting to note that since these excited multi-humped states emanate from the (already unstable) mixed state, they feature a relatively strong instability close to their bifurcation point.
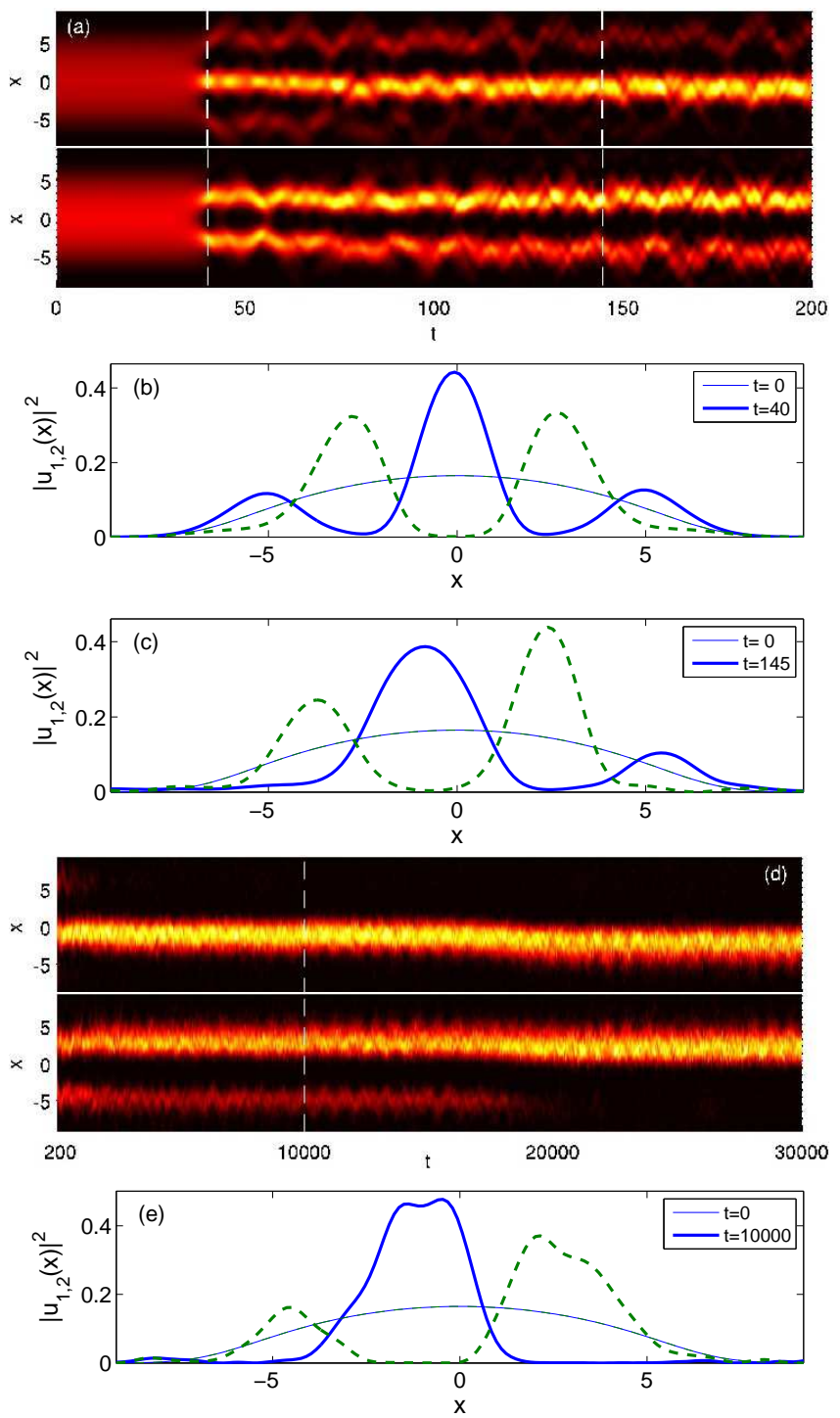

FIG. 14: (Color online) Dynamics of the mixed state for $g=5$ and $\Omega=0.2$. (a) Top and bottom subpanels correspond to the evolution of the densities for the first and second components, respectively, after applying an initial spatially random perturbation of size $1 \times 10^{-8}$. Panels (b) and (c) depict snapshots of the initial density and at the times indicated in panel (a) by the white vertical dashed lines. (d) Long term dynamics showing that the mixed state eventually approaches a separated state. (e) Snapshots of the densities at $t=0$ and $t=10,000$ (see white vertical dashed line in panel $(\mathrm{d})$ ).

\section{DYNAMICS OF UNSTABLE STATES}

In this section we present the dynamics of the different unstable states that were identified above. We start by analyzing the destabilization of the mixed state for a value of $g$ to the right of the bifurcation point A (see Fig. 11). In fact, for all the dynamical destabilization results presented in this section we chose a value of $g=5$ and $\Omega=0.2$ that is to the right of the bifurcation point 

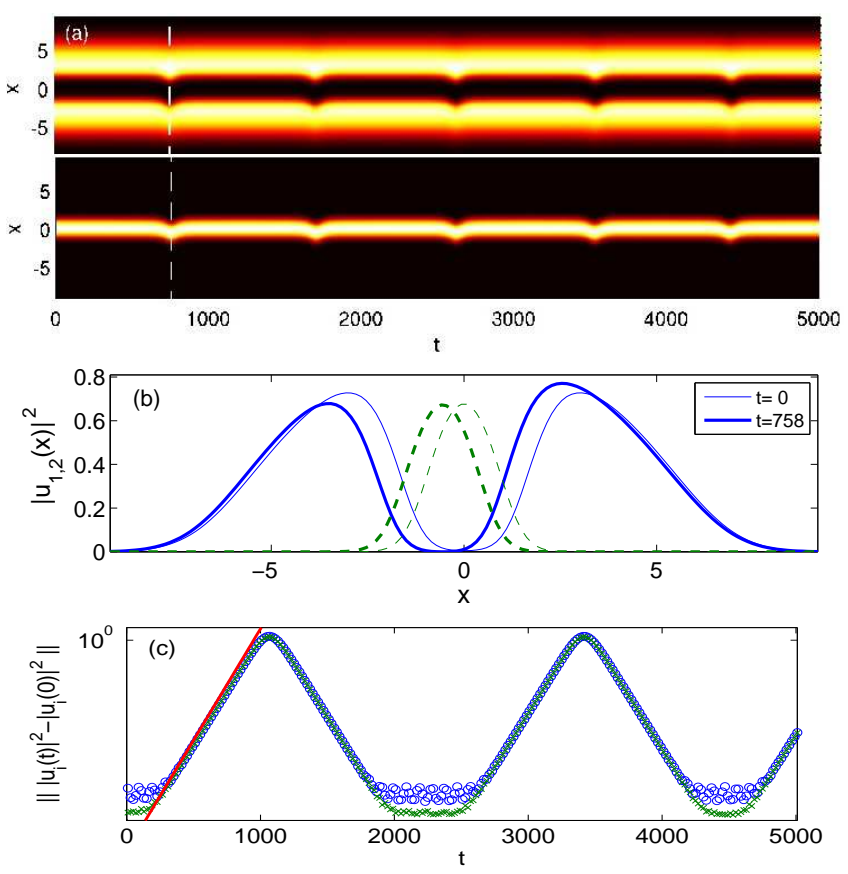

FIG. 15: (Color online) Dynamics of the unstable 1-2 hump state for $g=5$ and $\Omega=0.2$. (a) Same as in Fig. 14]a. (b) Snapshots of the densities at $t=0$ and $t=758$ (see white vertical dashed line in panel (a)). (c) Growth of the norm of the perturbation vs. time (in semi-log plot). Circles (crosses) correspond to the perturbation of the first (second) component and the red solid line is the instability growth obtained from the eigenvalue computation $(\max (\Re(\lambda)))=0.122)$.

E (see Fig. 11). Therefore, in this regime we have coexistence of several unstable multi-hump solutions and the stable phase-separated 1-1 hump state. Other parameter regions (not shown here) gave qualitatively the same results.

In Fig. 14 we show the destabilization of the mixed state. As it can be observed from the figure, the mixed state suffers an initial modulational instability that becomes apparent for $t>35$ that seeds a highly perturbed 2-3 hump solution [see panel (b)]. Since this new solution is also unstable for the chosen parameter values, it is rapidly converted into a relatively long lived 1-2 hump state [see panel (c)]. Nonetheless, as it is clear from the long term dynamics presented in panel (d), the 1-2 hump state, being unstable, eventually "decays" to the separated (1-1 hump) state. We use here the term "decaying" in quotes since our system is conservative (no dissipation) and thus there is no real decay.

In Fig. 15] we show the destabilization of the unstable 1-2 hump state. As it is obvious from the figure, although the instability eigenvalue is sizeable $\left[\lambda_{r}=0.122\right.$, see panel (c)]), the initially weak perturbation does not lead to the destruction of the 1-2 hump state. Instead, this unstable state just momentarily "jumps" to the left (or to the right, results not shown here), see panel (b),
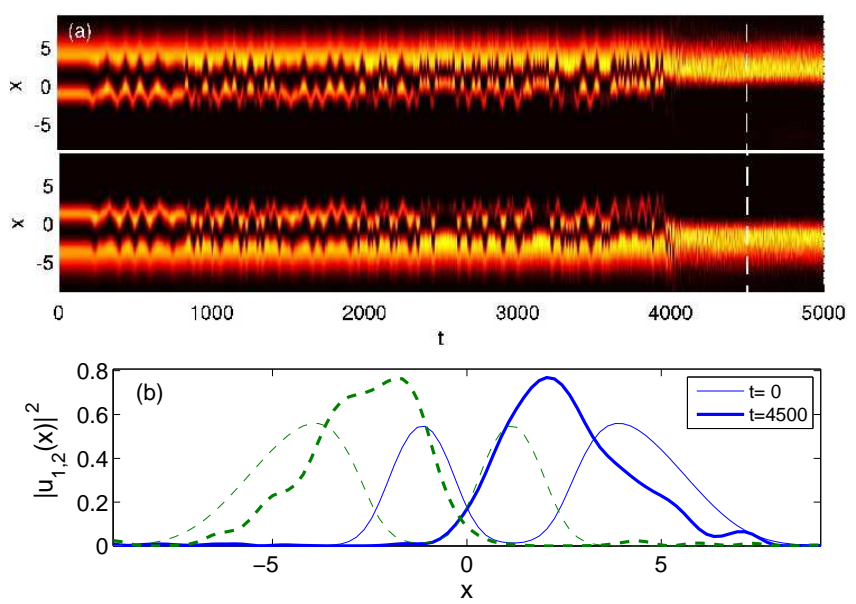

FIG. 16: (Color online) Dynamics of the unstable 2-2 hump state for $g=5$ and $\Omega=0.2$ as in Fig. 14. The eventual result of the instability is the formation of a robust and dynamically stable 1-1 hump state.
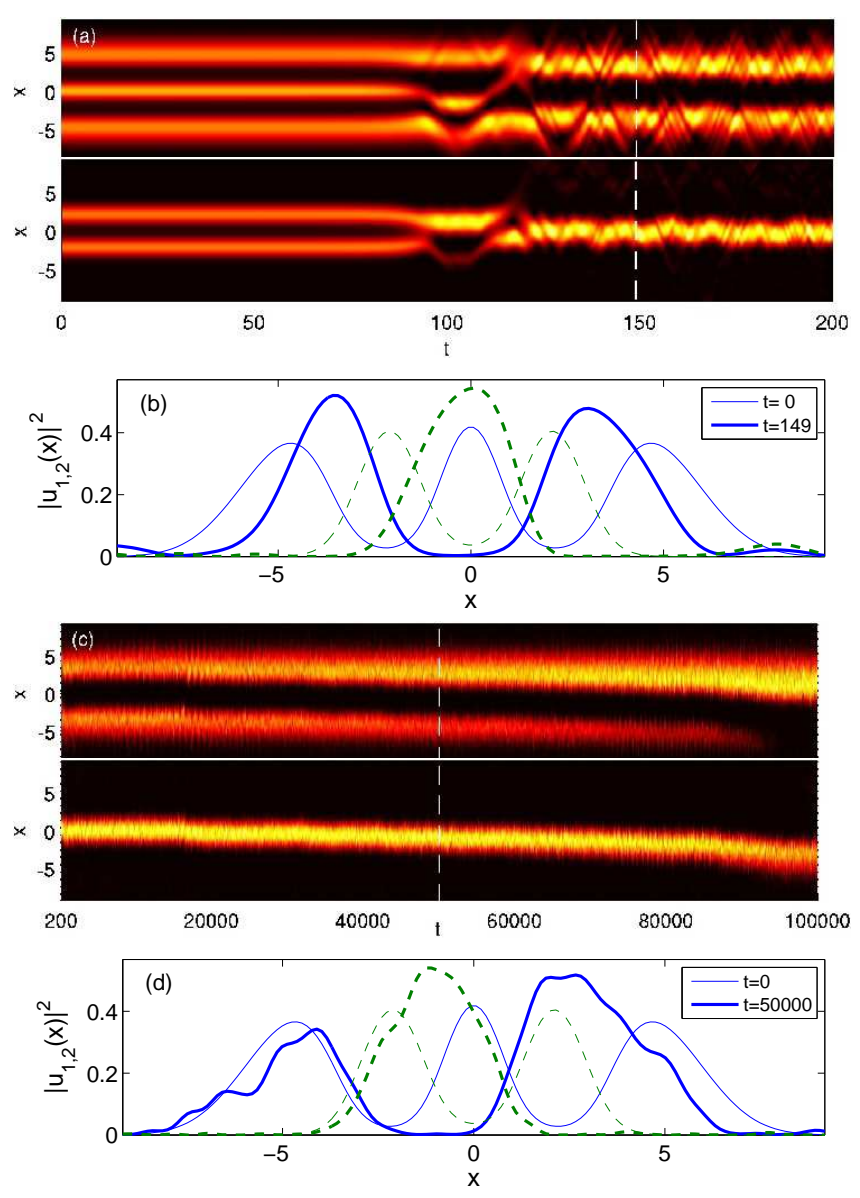

FIG. 17: (Color online) Dynamics of the unstable 2-3 hump state for $g=5$ and $\Omega=0.2$ as in Fig. 14. The initial instability leads to a transient 1-2 state that eventually approaches the separated state for extremely long propagation times $(t>100,000)$. 

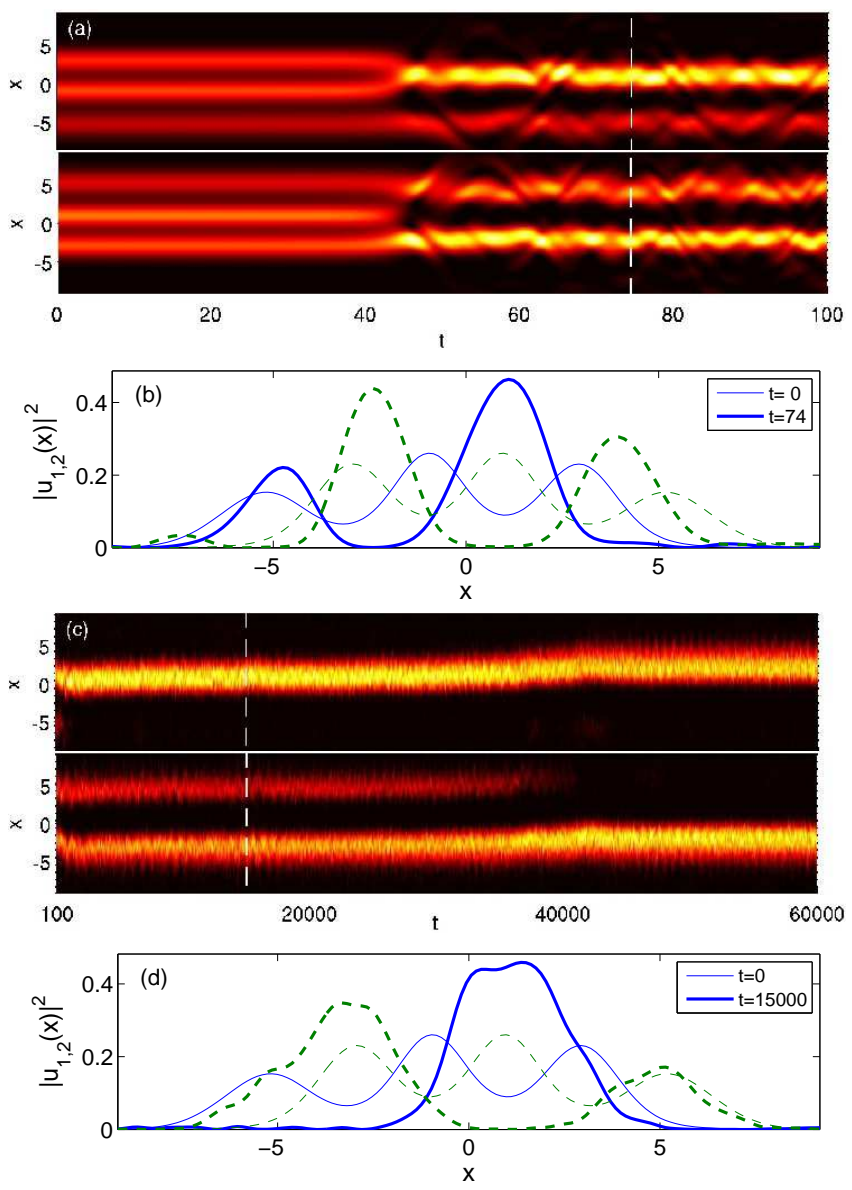

FIG. 18: (Color online) Dynamics of the unstable 3-3 hump state for $g=5$ and $\Omega=0.2$ as in Fig. 17 The initial instability dynamics reshapes the waveform into a $2-2$ state, then to long-lived transient a 1-2 state that eventually approaches the 1-1 state for $t>40,000$.

for a short period of time (becoming slightly asymmetric) and then comes back close to the 1-2 hump (symmetric) steady state configuration. This indicates that, for this parameter combination, the 1-2 hump state is a saddle fixed point. Thus, the orbit remains close to the steady 1-2 hump state and it is eventually "kicked out" along the unstable manifold. Then, it performs a quick excursion and "comes back" through the stable manifold.

In Fig. [16] we depict the destabilization dynamics of the 2-2 hump state. As it is evident from the figure, when compared to Fig. 15, the destabilization happens earlier $(t \approx 200)$ since the $2-2$ hump state is more unstable than the 1-2 hump state (see Fig. 13). As the dynamics of the perturbed 2-2 hump state evolves, it progressively favors phase separation until eventually the system rearranges itself into a 1-1 hump state at about $t \approx 4000$. Since the 1-1 hump is stable, this resulting configuration is sustained thereafter.

A similar scenario (fast initial destabilization, slow transient stage and eventual settling into the stable phase-separated state) is observed when following the dynamical destabilization of the 2-3 hump state. As it can be observed from Fig. 17, the relevant configuration destabilizes around $t \approx 90$ (earlier than its 1-2 and 22 hump counterparts given its larger instability growth rate [see Fig.13]). The dynamics goes through a transient 1-2 state and eventually settles into a highly perturbed separated state that is preserved thereafter as was the case also for the mixed state dynamics (see Fig. 14) and the 2-2 state (see Fig. 16) presented above.

Finally, in Fig. 18 we present the dynamical destabilization for the 3-3 hump state. Again, this state destabilizes even faster $(t=40)$ than the previous waveforms because of its higher instability eigenvalue (see Fig. 13). In this case, the 3-3 hump state first destabilizes into a highly perturbed $2-2$ state. Since the $2-2$ hump state is unstable, the ensuing dynamics results into a separated 1-2 hump state after $t>100$ which, in turn, eventually settles to a highly perturbed separated state.
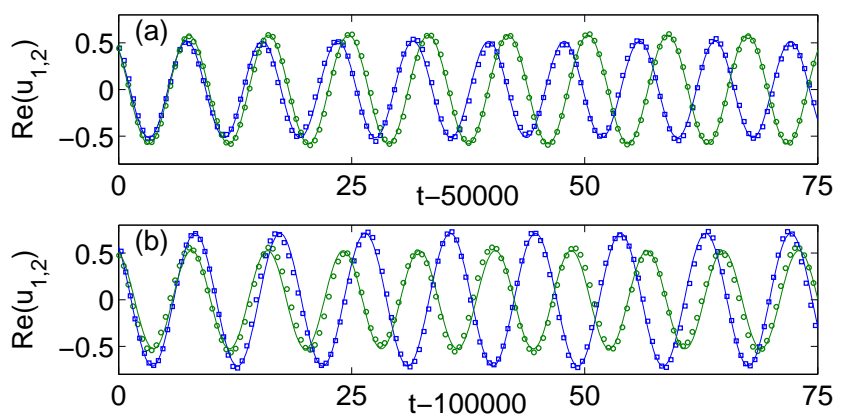

FIG. 19: (Color online) Dynamics of the real part of the wave functions for the 2-3 hump state of Fig. 17 for $g=5$ and $\Omega=0.2$. Panels (a) and (b) correspond, respectively, to times centered about $t=50,000$ and $t=100,000$. The blue square (green circles) correspond to the first (second) component. The continuous lines correspond to the best sinusoidal fit from which the local chemical potential can be extracted.

\section{EXISTENCE AND STABILITY OF ASYMMETRIC STATES}

It is interesting to observe in the above computations that the transient 1-2 hump state emanating from the destabilization of the mixed state (see Figs. 14) or from higher order states (see Figs. 17 and 18) has a relatively long life span. However, being this state also unstable, it eventually tends to a perturbed separated (i.e., 1-1 hump) state. The process of converting a 1-2 state into the separated state involves the effective shift of mass from one of the two humps (in the component with two humps) to the other one until all the mass is "swallowed up" by one hump resulting in a 1-1 hump. For example, as it can be observed in panel (d) of Fig. 17, at $t=50,000$ the right hump of the first component has 

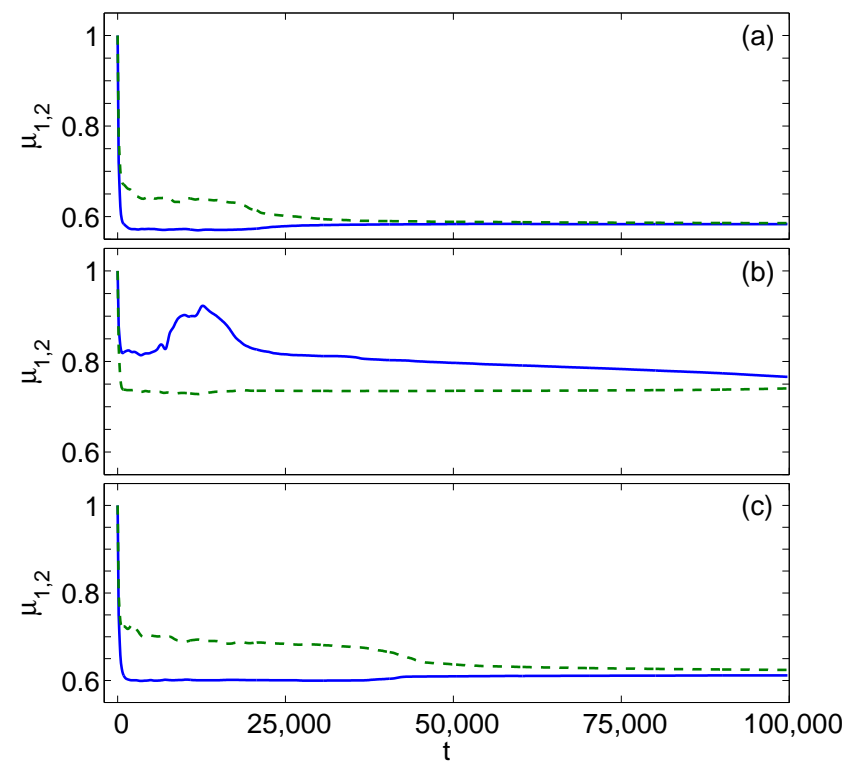

FIG. 20: (Color online) Local chemical potential at the location of maximum density for each component. Blue solid line corresponds to the first component while the green dashed line corresponds to the second component. The different panels correspond to the evolution of the local chemical potentials for the steady states: (a) mixed state (cf. Fig. 14), (b) 2-3 hump state (cf. Fig. 17), and (c) 3-3 hump states (cf. Fig. 18).

more mass than its left hump. Since the chemical potential (i.e., rotation frequency of the wave function in the complex plane) is closely related to the mass of the condensate, it is possible to follow the local change in mass by following the local chemical potential. In Fig. 19 we depict the oscillations of the real part of the wave functions at the location of the maximal density (see squares and circles in the figure). We then fit a sinusoidal curve (see solid lines in the figure) through the data to obtain the oscillation frequency. As it is obvious from the figure, although both components started with the 2-3 hump steady state with the same chemical potential $\mu_{1}=\mu_{2}=\mu=1$, the two components oscillate at different rates. In fact, at $t=50,000$ the local chemical potentials for each species is, respectively, $\mu_{1}=0.776$ and $\mu_{2}=0.734$, and at $t=100,000$ we have $\mu_{1}=0.688$ and $\mu_{2}=0.771$. We systematically extracted the local chemical potentials at the location of the maximum density for the mixed state, 2-3 hump state and 3-3 hump state and depict them in Fig. 20, As it can be observed from the figure, both chemical potentials start at $\mu_{1}=\mu_{2}=1$ but rapidly drop when the initial instability of the steady state develops for the three cases. Then, the chemical potentials slowly drift until they acquire values very close to each other at around the time when the dynamics settles to a perturbed phase separated state.

The fact that during the dynamical evolution of the unstable higher order states the local chemical potentials vary naturally prompts the question of existence and stability of steady states with different chemical potentials between the species. Up to this point all the steady states we analyzed supposed that both components had the same chemical potential. Namely, the evolution for each components is

$$
\begin{aligned}
& u_{1}(x, t)=w_{1}(x) e^{-i \mu_{1} t} \\
& u_{2}(x, t)=w_{2}(x) e^{-i \mu_{2} t},
\end{aligned}
$$

where the steady state profiles are $w_{1,2}(x)$ and $\mu_{1}=\mu_{2}=$ 1. We now relax this symmetric assumption and look for steady states with different chemical potentials between the components $\left(\mu_{1} \neq \mu_{2}\right)$. A full bifurcation diagram as a function of $\mu_{1}$, for fixed $\mu_{2}=1$, is depicted in Fig. 21. In this diagram we use in the vertical axis the difference in the variance of the steady state distribution between the two components. The different bifurcation branches correspond to the particular asymmetric states depicted in the surrounding insets. This diagram provides an account of how the different solutions bifurcate from each other. It is very interesting to follow the bifurcation path of all the states labeled from A to T during which all the higher order states are browsed continuously. Due to symmetry, a similar scenario is present when one keeps $\mu_{1}=1$ fixed and varies $\mu_{2}$ (results not shown here).

The stability in the bifurcation diagram depicted in Fig. 21 is denoted, as before, with a solid line for stable states and a dashed line for unstable states. As it can be noticed from the figures, as it was the case for symmetric states, all the asymmetric states are unstable except the mixed state. This is due to the fact that the interspecies coupling for these diagrams was chosen as $g=5$, i.e., high enough so that the mixed state in unstable. In fact, in Fig. 22 we show the largest real part of the eigenvalues for the different asymmetric states depicted in the bifurcation diagram in Fig. 21. As it was observed for the symmetric case, the higher the order of the asymmetric state the more unstable it becomes. It is crucial to note that the 1-2 hump state seems to get stabilized for $\mu_{2}=1$ and $\mu_{1}>1.12$ (see top panel of Fig. 22). However, after close inspection, see the bottom panel in Fig. 22, the real part of the eigenvalue for the 1-2 hump state never vanishes but instead becomes extremely small (between $10^{-8}$ and $10^{-2}$ ) until the branch disappears (at $\mu_{1} \approx 1.65$ for $\left.\mu_{2}=1\right)$. This very weak instability explains the fact why the transient 1-2 hump state appears to be sustained for very long times in the evolutions depicted in Figs. 14, 17. and 18

\section{CONCLUSIONS}

In the present work, we have analyzed the emergence of non-topological, phase-separated states in the immiscible regime out of mixed ones in the miscible regimes, as a natural parameter of the system (namely the interspecies interaction strength) was varied. Our analysis 


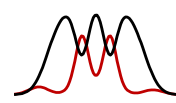

A
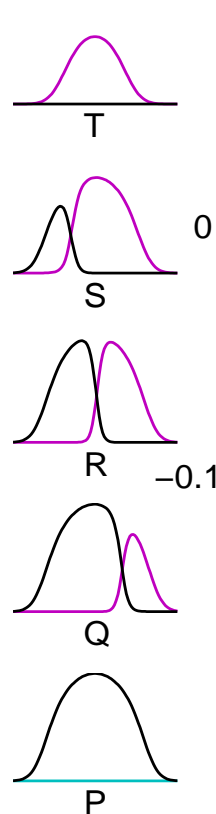

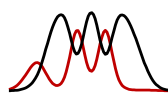

$\mathrm{B}$

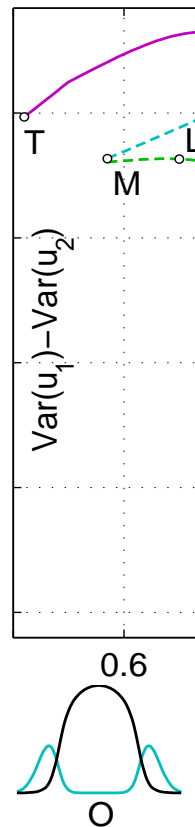

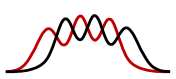

C

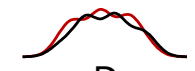

$\mathrm{D}$

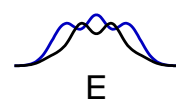

$\mathrm{E}$
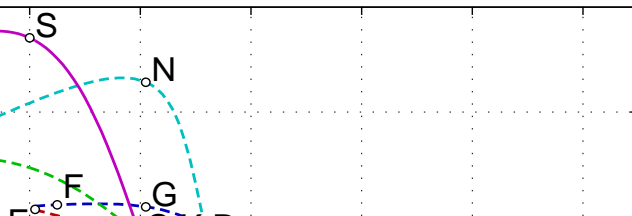
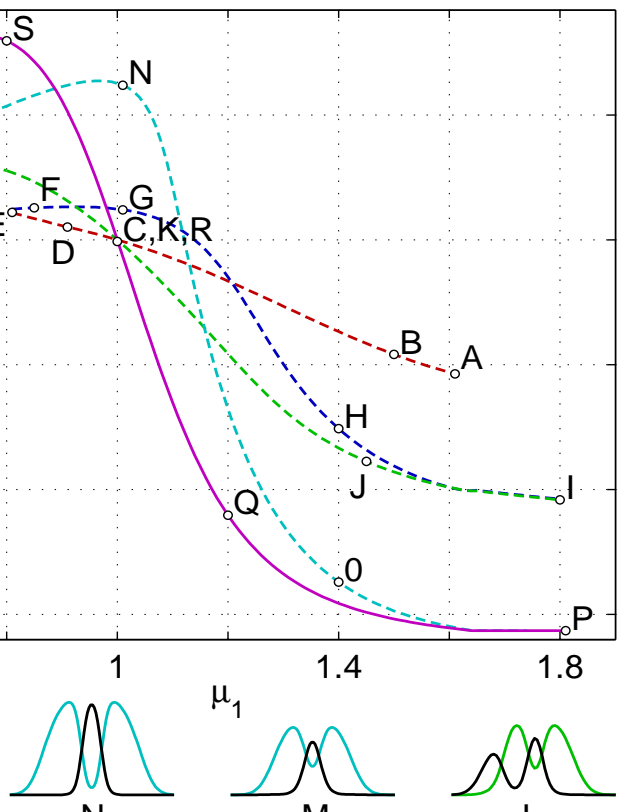

$\mathrm{N}$ $\mu_{1}$

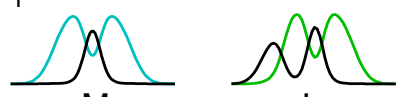

$\mathrm{L}$

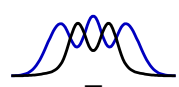

$\mathrm{F}$
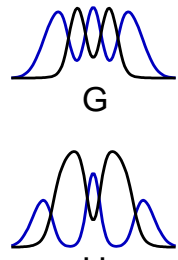

$\mathrm{H}$
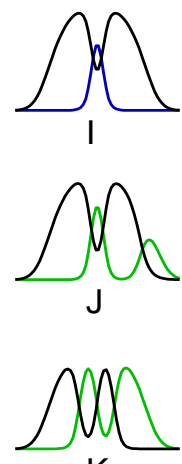

$\mathrm{K}$

FIG. 21: (Color online) Bifurcation diagram of asymmetric states for constant $\mu_{2}=1$ as a function of $\mu_{1}$. The vertical axis corresponds to the difference of the variance of the steady state configurations for both components for $g=5$ and $\Omega=0.2$. Stable (unstable) solutions branches are depicted with black solid (dashed) lines. Typical solutions for each branch are depicted in the surrounding insets.
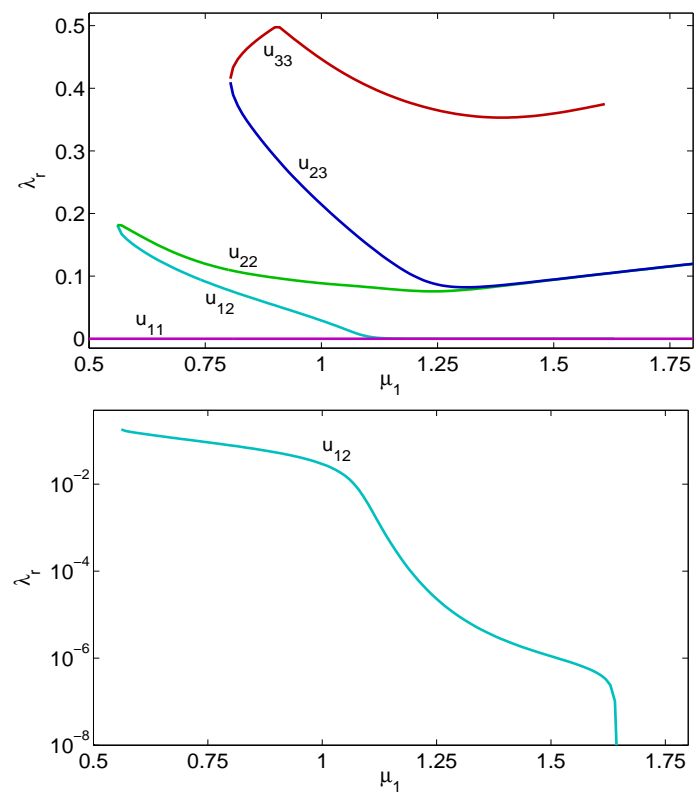

FIG. 22: (Color online) Real part of largest eigenvalue for the asymmetric mixed states as a function of $\mu_{1}$ with $\mu_{2}=1$, $g=5$ and $\Omega=0.2$. The bottom panel corresponds to the eigenvalue for the 1-2 hump state plotted in logarithmic scale. was presented for the case of magnetically trapped twocomponent Bose-Einstein condensates i.e., two incoherently coupled NLS equations with a parabolic potential. Using a variational approach, we obtain a reduced model describing the statics, stability and dynamics of each condensate cloud. We are able to elucidate the miscibility boundary for the interspecies coupling parameter as the strength of the magnetic trap (and/or the chemical potential of the system) is varied. The approach is also capable of accurately capturing the spatial oscillations of the clouds about the stable stationary states (both for mixed and for phase-separated states). In particular, for relatively small interspecies coupling, the two condensed clouds do not phase-separate (mixed state) giving rise, if the BEC clouds are initially displaced with respect to each other, to oscillations through each other. On the other hand, for relatively large interspecies coupling, the two clouds form a stable phase-separated state which can entertain oscillations about the equilibrium separation between the components. A further dynamical reduction allows to understand this behavior more intuitively based on an effective potential that undergoes a bifurcation from a single well (mixed state) to a double well (phase-separated state) form as the interspecies coupling parameter is increased. We also describe the bifurcation scenario of higher order phase-separated states, 
as the interspecies coupling parameter is increased. We observe that several (interwoven between the two components) bands of density modulations progressively arise out of the mixed states giving rise to higher excited states. Among all the phase-separated states, only the first excited one with one hump in each component is found to be dynamically stable for all values of the interspecies interaction strength past its bifurcation point. We furthered our analysis by studying the existence and stability of asymmetric states for which the chemical potentials for each species is different. We found, similar to the symmetric case of equal chemical potentials, that the only stable steady state for high enough interspecies coupling is the separated state. Nonetheless, we found that in some regimes, the (asymmetric variant of the) state with one band in one component surrounded by two bands of the other component (the 1-2 hump state) can have an extremely weak instability and thus facilitating its potential observability in numerical experiments.

There are numerous directions along which the present work can be naturally extended. For example, within the one-dimensional context, it is natural to seek to relax the simplifying assumption of the intraspecies scattering lengths (and by extension the self-interaction coefficients of the two components, $g_{11}$ and $g_{22}$ ) being equal. However, this extension will unfortunately have to come at the expense of an ansatz with different amplitude, width, etc. parameters between the components, which will render the intuitive and explicit analytical results obtained herein much more tedious.

Another natural extension is to try to generalize the ideas presented herein into higher-dimensional or larger number of component (i.e., spinor) settings. Especially in the former case, more complicated waveforms such as crosses and propellers have been predicted in twocomponent condensates in two-dimensions [40] and it would be particularly interesting to examine whether these, as well as more complicated multi-hump waveforms emerge systematically from the corresponding mixed state via two-dimensional generalizations of the bifurcations presented herein. Such studies are presently in progress and will be presented in future publications.

\section{Acknowledgments}

The authors acknowledge support from NSF-DMS0806762. PGK also acknowledges support from NSFDMS-0349023 (NSF-CAREER) and from the Alexander von Humboldt Foundation.
[1] C. J. Pethick and H. S. Smith, Bose-Einstein Condensation in Dilute Gases, Cambridge University Press, Cambridge, 2002.

[2] L.P. Pitaevskii and S. Stringari, Bose-Einstein Condensation, Oxford University Press (Oxford, 2003).

[3] P.G. Kevrekidis, D.J. Frantzeskakis, and R. CarreteroGonzález (eds.), Emergent nonlinear phenomena in Bose-Einstein condensates. Theory and experiment (Springer-Verlag, Berlin, 2008).

[4] Yu.S. Kivshar and G.P. Agrawal, Optical solitons: from fibers to photonic crystals, Academic Press (San Diego, 2003).

[5] T.-L. Ho and V.B. Shenoy, Phys. Rev. Lett. 77, 3276 (1996); H. Pu and N.P. Bigelow, Phys. Rev. Lett. 80, 1130 (1998).

[6] B.D. Esry et al., Phys. Rev. Lett. 78, 3594 (1997).

[7] Th. Busch et al., Phys. Rev. A 56, 2978 (1997); R. Graham and D. Walls, Phys. Rev. A 57, 484 (1998); H. $\mathrm{Pu}$ and N.P. Bigelow, Phys. Rev. Lett. 80, 1134 (1998); B.D. Esry and C.H. Greene, Phys. Rev. A 57, 1265 (1998).

[8] M. Trippenbach, K. Goral, K. Rzazewski, B. Malomed, and Y.B. Band, J. Phys. B 33, 4017 (2000).

[9] I.M. Merhasin, B. A. Malomed, R. Driben, J. Phys. B: At. Mol. Opt. Phys. 38, 877-892 (2005).

[10] I.M. Merhasin, B.A. Malomed and R. Driben, Phys. Scripta T116, 18 (2006).

[11] S. Coen and M. Haelterman, Phys. Rev. Lett. 87, 140401 (2001).

[12] P. Öhberg and L. Santos, Phys. Rev. Lett. 86, 2918 (2001).
[13] Th. Busch and J.R. Anglin, Phys. Rev. Lett. 87, 010401 (2001).

[14] C. Becker, S. Stellmer, S. Soltan-Panahi, S. Dorcher, M. Baumert, E.-M. Richter, J. Kronjager, K. Bongs and K. Sengstock, Nature Phys. 4, 496 (2008).

[15] K. Kasamatsu, M. Tsubota and M. Ueda, Int. J. Mod. Phys. B 19, 1835 (2005).

[16] B. Deconinck, J.N. Kutz, M.S. Patterson and B.W. Warner, J. Phys. A 36, 5431 (2003).

[17] C.J. Myatt, E.A. Burt, R.W. Ghrist, E.A. Cornell, and C.E. Wieman Phys. Rev. Lett. 78, 586 (1997).

[18] D.S. Hall, M.R. Matthews, J.R. Ensher, C.E. Wieman, and E.A. Cornell, Phys. Rev. Lett. 81, 1539 (1998).

[19] D.M. Stamper-Kurn, M. R. Andrews, A. P. Chikkatur, S. Inouye, H.-J. Miesner, J. Stenger, and W. Ketterle, Phys. Rev. Lett. 80, 2027 (1998).

[20] G. Modugno, G. Ferrari, G. Roati, R.J. Brecha, A. Simoni, M. Inguscio, Science 294, 1320 (2001).

[21] M. Mudrich, S. Kraft, K. Singer, R. Grimm, A. Mosk, and M. Weidemüller, Phys. Rev. Lett. 88, 253001 (2002).

[22] V. Schweikhard, I. Coddington, P. Engels, S. Tung and E.A. Cornell, Phys. Rev. Lett. 93, 210403 (2004).

[23] K.M. Mertes, J.W. Merrill, R. Carretero-González, D.J. Frantzeskakis, P.G. Kevrekidis and D.S. Hall, Phys. Rev. Lett. 99, 190402 (2007).

[24] S.B. Papp, J.M. Pino and C.E. Wieman, Phys. Rev. Lett. 101, 040402 (2008).

[25] K. Kasamatsu, and M. Tsubota, Phys. Rev. A 74, 013617 (2006).

[26] G. Karali, P.G. Kevrekidis and N.K. Efremidis, J. Phys. A: Math. Theor 42, 045206 (2009). 
[27] R. Carretero-González, D.J. Frantzeskakis, and P.G. Kevrekidis, Nonlinearity 21, R139 (2008).

[28] M.-S. Chang, C.D. Hamley, M.D. Barrett, J.A. Sauer, K.M. Fortier, W. Zhang, L. You, and M.S. Chapman, Phys. Rev. Lett. 92, 140403 (2004).

[29] J. Stenger, S. Inouye, D.M. Stamper-Kurn, H.-J. Miesner, A.P. Chikkatur, and W. Ketterle. Nature 396, 345 (1998).

[30] H.E. Nistazakis, D.J. Frantzeskakis, P.G. Kevrekidis, B.A. Malomed, R. Carretero-González, and A.R. Bishop, Phys. Rev. A 76, 063603 (2007).

[31] A.E. Leanhardt, Y. Shin, D. Kielpinski, D.E. Pritchard, and W. Ketterle, Phys. Rev. Lett. 90, 140403 (2003).

[32] J. Ieda, T. Miyakawa, and M. Wadati, Phys. Rev. Lett. 93, 194102 (2004).

[33] J. Ieda, T. Miyakawa, and M. Wadati, J. Phys. Soc. Jpn. 73, 2996 (2004).
[34] L. Li, Z. Li, B.A. Malomed, D. Mihalache, and W.M. Liu, Phys. Rev. A 72, 033611 (2005).

[35] W. Zhang, Ö.E. Müstecaplioglu, and L. You, Phys. Rev. A 75, 043601 (2007).

[36] M. Uchiyama, J. Ieda, and M. Wadati, J. Phys. Soc. Jpn. 75, 064002 (2006).

[37] B.J. Dabrowska-Wüster, E.A. Ostrovskaya, T.J. Alexander, and Y.S. Kivshar, Phys. Rev. A 75, 023617 (2007).

[38] H.E. Nistazakis, D.J. Frantzeskakis, P.G. Kevrekidis, B.A. Malomed, and R. Carretero-González, Phys. Rev. A 77, 033612 (2008).

[39] A. Weller, J.P. Ronzheimer, C. Gross, J. Esteve, M.K. Oberthaler, D.J. Frantzeskakis, G. Theocharis, and P.G. Kevrekidis, Phys. Rev. Lett. 101, 130401 (2008).

[40] B.A. Malomed, H.E. Nistazakis, D.J. Frantzeskakis, and P.G. Kevrekidis, Phys. Rev. A 70, 043616 (2004). 